Review

\title{
Emotional Prosody Processing in Schizophrenic Patients: A Selective Review and Meta-Analysis
}

\author{
Yi Lin ${ }^{1}$, Hongwei Ding ${ }^{1, *}$ and Yang Zhang ${ }^{2, *}$ \\ 1 Institute of Cross-Linguistic Processing and Cognition, School of Foreign Languages, Shanghai Jiao Tong \\ University, Shanghai 200240, China; carol.y.lin@sjtu.edu.cn \\ 2 Department of Speech-Language-Hearing Sciences \& Center for Neurobehavioral Development, \\ University of Minnesota, Twin Cities, MN 55455, USA \\ * $\quad$ Correspondence: hwding@sjtu.edu.cn (H.D.); zhanglab@umn.edu (Y.Z.); Tel.: +86-213420-5377 (H.D.); \\ +1-612-624-7818 (Y.Z.)
}

Received: 13 August 2018; Accepted: 14 October 2018; Published: 17 October 2018

\begin{abstract}
Emotional prosody (EP) has been increasingly recognized as an important area of schizophrenic patients' dysfunctions in their language use and social communication. The present review aims to provide an updated synopsis on emotional prosody processing (EPP) in schizophrenic disorders, with a specific focus on performance characteristics, the influential factors and underlying neural mechanisms. A literature search up to 2018 was conducted with online databases, and final selections were limited to empirical studies which investigated the prosodic processing of at least one of the six basic emotions in patients with a clear diagnosis of schizophrenia without co-morbid diseases. A narrative synthesis was performed, covering the range of research topics, task paradigms, stimulus presentation, study populations and statistical power with a quantitative meta-analytic approach in Comprehensive Meta-Analysis Version 2.0. Study outcomes indicated that schizophrenic patients' EPP deficits were consistently observed across studies $(d=-0.92$, $95 \% \mathrm{CI}=-1.06<\delta<-0.78)$, with identification tasks $(d=-0.95,95 \% \mathrm{CI}=-1.11<\delta<-0.80)$ being more difficult to process than discrimination tasks $(d=-0.74,95 \% \mathrm{CI}=-1.03<\delta<-0.44)$ and emotional stimuli being more difficult than neutral stimuli. Patients' performance was influenced by both participant- and experiment-related factors. Their social cognitive deficits in EP could be further explained by right-lateralized impairments and abnormalities in primary auditory cortex, medial prefrontal cortex and auditory-insula connectivity. The data pointed to impaired pre-attentive and attentive processes, both of which played important roles in the abnormal EPP in the schizophrenic population. The current selective review and meta-analysis support the clinical advocacy of including EP in early diagnosis and rehabilitation in the general framework of social cognition and neurocognition deficits in schizophrenic disorders. Future cross-sectional and longitudinal studies are further suggested to investigate schizophrenic patients' perception and production of EP in different languages and cultures, modality forms and neuro-cognitive domains.
\end{abstract}

Keywords: emotional prosody processing; schizophrenia; meta-analysis

\section{Introduction}

Schizophrenia is a severe chronic psychiatric illness affecting people's educational, vocational and daily performance [1]. More than 21 million people worldwide suffer from this mental disorder, with males (12 million) more common than females (9 million). People with schizophrenia often bear profound dysfunctions in social cognition, which refers to how they think, feel and behave, including the sense of self [2,3]. They are also characterized by distortions in emotions and language [4], which usually result in an isolation in social interactions [5]. 
Social cognition research has four characteristic features, respectively focusing on mentalism, attempting to understand the causal mechanism of the cognition process, adopting approaches and ideas from multiple disciplines, and concerning with real-world applications [6]. Recent years have witnessed increasing attention on the study of social cognition deficits in clinical populations, including patients with schizophrenia. These studies largely fall into three thematic categories: (1) Theory of Mind, which investigates how participants represent their mental states and make inferences about others' intentions, (2) emotion perception, which addresses how participants identify and discriminate facial and vocal affect as well as other social signals such as gesture, and (3) attributional style, which is concerned with how participants give explanations to the positive and negative events happening in their lives [6,7]. A line of studies has investigated how social cognition abnormality is related to other domains in schizophrenic patients, such as neurobiology [7], neurocognition [8], functional outcomes [8,9] and negative symptoms [6]. There is a growing amount of evidence showing schizophrenic patients' impairments in social cognition, which can be regarded as a powerful predictor of patients' impaired social functioning $[7,10]$. Some researchers further identified comparable social cognition disorders between schizophrenic patients and patients with other psychotic disorders such as bipolar disorder [11], and highlighted the importance of continuous psychosocial support and social cognition analysis techniques and training programs [12-14].

Successful interpersonal communication requires an individual to be able to interpret and respond to pertinent social signals of others in dynamic contexts. As a key domain of social cognition and an organic integration of emotions and language, emotional prosody (EP), also known as affective prosody, is an important component of communication conveying speakers' feelings and intent through variations of parameters such as pitch, stress and duration [15]. Emotional prosody processing (EPP) refers to the perception and production of EP, the study of which can contribute to the clinical diagnosis and treatment as well as in-depth research on participants' processing of both linguistic and non-linguistic information in communication. The EPP performance of patients with psychotic disorders have been a focal point in recent years' research, involving people suffering from schizophrenia, borderline personality disorder, autism spectrum disorder, bipolar disorder, depression, and schizoaffective disorder [11,16-19]. In these studies, affect perception ability was shown to have an association with social functioning and play an increasingly important role in interpersonal relations.

To date, a number of studies have discovered that people with schizophrenia show reduced emotional expressivity and impaired emotional prosodic comprehension [20-22]. Although a complete inability of EPP in terms of the perceptual, cognitive and social functions is unlikely in these individuals, the observed differences in both areas of EP comprehension and production deserve systematic exploration to determine their functional and clinical significance, which may lead to the development of novel and alternative ways to approach social cognition deficits in schizophrenic patients and patients with psychiatric disorders in general. Considering that emotion is a significant component of social cognition, schizophrenic patients' deficits at this level may result in more interpersonal misunderstanding or inappropriate social behaviors [23], thus preventing them from assimilating into a larger social community.

One challenging problem that faces the field of schizophrenia research and clinical practice is that although cognitive impairment generally co-occurs with psychosis, it may reflect processes or an epiphenomenon independent of genetic and biological factors that lead to the mental disorder itself. In terms of EPP, it remains unclear whether there is sufficient and strong evidence to determine EPP impairment as a pervasive and intrinsic defining feature of the psychopathology of schizophrenia. To our knowledge, few studies have systematically examined schizophrenic patients' EPP except a meta-analysis that only focused on behavioral studies undertaken between 1980 and 2007. The scope and depth of that review are limited as it did not take into account recent advancements using new experimental techniques and did not evaluate the influence of different task paradigms [24]. With the advancement of neuroimaging techniques, it is of great necessity to integrate or reconcile findings from the latest brain research studies with behavioral studies under different experimental designs in 
order to gain a better understanding of schizophrenic patients' EPP deficits, including heterogeneity in performance and the underlying neural markers. Presumably, in the case of EPP, various dissociable component behavioral elements can be examined, including input-related attentional and cognitive processes and output-related motor control processes, to identify potential specific target components for therapeutic interventions. However, there is still a lack of a comprehensive overview to examine the additive and integrative nature of these components in this regard. In particular, a synopsis of studies is in need to summarize (a) the central topics, research design and statistical analysis of the latest related studies, (b) schizophrenic patients' EPP performance under different experimental conditions influenced by variations in task paradigms, response options, stimulus modalities, participants' demographic, clinical and cognitive backgrounds, and (c) the physiological and neuroanatomical basis in terms of hemispheric, spatial and temporal processing of EPP in patients with schizophrenia.

As methods of cognitive and functional assessment are fundamental to interpretations of the research findings on schizophrenia, it is necessary to periodically reflect upon the new trends of developments and existing tools and protocols that may need modifications to further advance the field. By analyzing selected behavioral and neuroscientific studies via five major online databases, our current selective review aims to (a) summarize the characteristics of the latest studies, (b) determine the magnitude of impairments in EPP of schizophrenic patients by calculating the effect size in terms of different task paradigms, and (c) identify the potential factors and neural mechanisms influencing schizophrenic patients' EPP performance. We hope to deepen the understanding of the nature of EPP impairments in schizophrenic patients as a core deficit and contribute to the diagnosis and rehabilitation of this psychiatric disorder. This review also serves to provide suggestions and implications for future research on this promising topic for experts from multidisciplinary areas, including speech and language, psychotic disorder, psychosocial mediation, and neuroimaging.

\section{Method}

\subsection{Inclusion and Exclusion Criteria}

\subsubsection{Types of Studies}

The studies eligible for inclusion in this review had to investigate EPP of patients with schizophrenia in comparison with a control group(s). The following types of studies were included:

(a) cross-sectional studies comparing schizophrenic patients versus healthy controls or/and versus a clinical comparison group (i.e., patients with a different psychiatric disorder or neurological condition);

(b) longitudinal studies comparing participants' performance before versus after schizophrenia.

We only included studies containing at least one type of the six basic emotions classified by Ekman [25]: anger, disgust, fear, happiness, sadness and surprise. Complex or social emotions (such as contempt, sarcasm, trustworthiness, and embarrassment) were therefore not taken into consideration in this review. The studies needed to use behavioral tasks such as identification, discrimination, labeling and rating. In these tasks, participants were required to evaluate the stimuli based on the emotional information conveyed by the stimuli. The stimuli could be of any form (e.g., words, sentences, pictures, sounds) and of any modality (e.g., auditory, visual). Studies exclusively focusing on mood, emotional memory, emotional production and facial emotional processing were excluded. Finally, reviews, editorials, conceptual papers and book chapters with no original data were excluded, so were the abstracts (primarily conference papers) with no access to full texts.

\subsubsection{Types of Participants}

Given that schizophrenia typically begins in early adulthood or late adolescence, studies that involved adult patient populations (over 18 years old) were eligible for this review. Participants must have a clear diagnosis of schizophrenia through Diagnostic and Statistical Manual of Mental Disorders 
(DSM) or WHO International Classification of Disorders (ICD). We also excluded studies involving participants with co-morbid physical or psychological diseases, uncorrected auditory or visual impairments or any neurocognitive disorders. Studies concerning schizoaffective disorders were included whereas schizotypal personality disorders (STPD) were not taken for review since the classification of STPD has not been unified in DSM-V and ICD-10 [26]. The current review also excluded prodromal schizophrenia patients so that we can provide a more accurate description of people suffering from schizophrenia at the time of the experiments. Additionally, demographic information such as gender, education, the duration and severity of illness evaluated by PANSS (Positive and Negative Syndrome Scale) should be specified as detailed as possible [27]. The ramifications of our inclusion and exclusion criteria for this selective review are further elaborated in the discussion section.

\subsubsection{Types of Measures}

Studies must adopt an experimental or a quasi-experimental method and report a quantitative measure of EPP.

\subsection{Searching Strategies for Identification of Studies}

Comprehensive research was conducted electronically through the following databases: Web of Science Core Collection, MEDLINE, PsycINFO, PsycARTICLES and Psychology and Behavioral Sciences Collection. The first two databases were retrieved from Web of Science (WOS) while the rest were accessed using EBSCO Academic Source Complete. The advanced search keywords were "emotional prosody processing" OR "affective prosody processing" AND "schizophrenia". The search included studies with publication dates up to April 2018. For each study included in the review, we also conducted manual searches of reference lists so as to identify additional potential studies.

\subsection{Data Collection and Analysis}

\subsubsection{Selection of Studies}

Initial searches in the databases returned a total of 489 studies. The title and abstract of each study were first checked in accordance with the inclusion and exclusion criteria, which resulted in 46 candidates after removing duplicated citations across the search results. The full text of these 46 papers was then examined, and another 5 studies were excluded after detailed checking (see Table 1 for reasons for exclusion). The screening process discovered 23 studies that fully met our selection criteria. After reading the full contents in detail, six additional studies were identified from the reference lists in the 23 articles. Thus, the total number of the selected publications for the final meta-analysis is 29. The flowchart of study selection is presented in Figure 1.

\subsubsection{Data Extraction}

Data were extracted concerning the following key elements: (a) general characteristics of the studies, (b) statistical analysis and (c) study outcome. We designed a data collection form to record a range of aspects, including study type (behavioral or neurological), sample size, stimulus presentation, task paradigm, response option, demographic information, methods of analysis, reporting of statistical results, and major findings. 
Table 1. Characteristics of studies related to emotional prosody processing (EPP) in schizophrenia.

\begin{tabular}{|c|c|c|c|c|c|c|c|c|c|c|c|c|c|}
\hline \multirow[b]{2}{*}{ Year } & \multirow[b]{2}{*}{ First Author } & \multirow{2}{*}{$\begin{array}{c}\text { Study Type } \\
\text { (Neurological } \\
\text { Technique): Study } \\
\text { Topic }\end{array}$} & \multirow[b]{2}{*}{$\begin{array}{c}\text { Country } \\
\text { Language }\end{array}$} & \multirow[b]{2}{*}{ Task Paradigm } & \multirow[b]{2}{*}{$\begin{array}{l}\text { Stimulus Modality: } \\
\text { Stimulus Form }\end{array}$} & \multirow[b]{2}{*}{$\begin{array}{c}\text { Response } \\
\text { Option }\end{array}$} & \multicolumn{5}{|c|}{ Patient Information } & \multirow[b]{2}{*}{$\begin{array}{c}\text { Statistical } \\
\text { Analysis Methods }\end{array}$} & \multirow[b]{2}{*}{$\begin{array}{l}\text { Notes (Reasons for } \\
\text { Exclusion from } \\
\text { Meta-Analysis) }\end{array}$} \\
\hline & & & & & & & $\begin{array}{l}\text { Number } \\
(\text { Male\%) }\end{array}$ & $\begin{array}{l}\text { Age } \\
\text { (S.D.) }\end{array}$ & $\begin{array}{l}\text { Education } \\
\text { (years) }\end{array}$ & IQ & $\begin{array}{l}\text { Illness } \\
\text { Duration } \\
\text { (years) }\end{array}$ & & \\
\hline 2001 & Ross [28] & $\begin{array}{l}\text { Behavioral: } \\
\text { Hemispheric } \\
\text { dysfunction in } \\
\text { schizophrenia } \\
\text { patients and the } \\
\text { neurology of EPP }\end{array}$ & $\begin{array}{l}\text { the US } \\
\text { English }\end{array}$ & $\begin{array}{c}\text { Identification } \\
\text { (SSC and SPP) } \\
\text { Discrimination } \\
\text { (SSC pair) }\end{array}$ & $\begin{array}{c}\text { A: sentences, } \\
\text { monosyllables, words } \\
\text { and asyllabic sounds }\end{array}$ & C & $45(87 \%)$ & $\begin{array}{l}42.5 \\
(7.2)\end{array}$ & $\begin{array}{l}13.8 \\
(2.3)\end{array}$ & N/A & N/A & $\begin{array}{l}\text { ANOVA, } \\
\text { Chi-square test } \\
\text { and PCA }\end{array}$ & $\begin{array}{l}\text { Excluded from the } \\
\text { meta-analysis of } \\
\text { identification and } \\
\text { discrimination task } \\
\text { but included in the } \\
\text { overall effect size } \\
\text { calculation (only } \\
\text { reporting the results } \\
\text { for comprehension) }\end{array}$ \\
\hline 2002 & Hooker [29] & $\begin{array}{l}\text { Behavioral: EPP } \\
\text { and social } \\
\text { functioning }\end{array}$ & $\begin{array}{l}\text { the US } \\
\text { English }\end{array}$ & $\begin{array}{l}\text { Identification } \\
\text { (SSC) }\end{array}$ & $\begin{array}{c}\text { A: sentences, } \\
\text { V: facial pictures }\end{array}$ & C & $20(75 \%)$ & $\begin{array}{l}39.3 \\
(8.5)\end{array}$ & $\begin{array}{l}12.7 \\
(2.9)\end{array}$ & N/A & $\begin{array}{l}18.8 \\
(10.2)\end{array}$ & ANOVA & \\
\hline 2005 & Leitman [30] & $\begin{array}{l}\text { Behavioral: } \\
\text { Impaired EPP and } \\
\text { basic auditory } \\
\text { processing deficits }\end{array}$ & $\begin{array}{l}\text { the US } \\
\text { English }\end{array}$ & $\begin{array}{l}\text { Identification } \\
\text { (SSC) } \\
\text { Discrimination } \\
\text { (SSC pair) }\end{array}$ & $\begin{array}{l}\text { A: sentences, } \\
\text { V: facial pictures }\end{array}$ & C & $43(77 \%)$ & $\begin{array}{l}39.0 \\
(12.0)\end{array}$ & $\begin{array}{l}10.6 \\
(3.2)\end{array}$ & N/A & $\begin{array}{l}17.4 \\
(9.6)\end{array}$ & $\begin{array}{l}\text { ANOVA, } \\
\text { Spearman and } \\
\text { PCA }\end{array}$ & \\
\hline \multirow[t]{2}{*}{2005} & \multirow[t]{2}{*}{ Rossell [31] } & \multirow{2}{*}{$\begin{array}{c}\text { Behavioral: } \\
\text { Impaired EPP and } \\
\text { auditory-verbal } \\
\text { hallucinations }\end{array}$} & \multirow{2}{*}{$\begin{array}{l}\text { Australia } \\
\text { English }\end{array}$} & \multirow{2}{*}{$\begin{array}{c}\text { Identification } \\
\text { (SSC) } \\
\text { Discrimination } \\
\text { (SSC pair) } \\
\end{array}$} & \multirow{2}{*}{$\begin{array}{l}\text { A: sentences, words, } \\
\text { non-words, syllables } \\
\text { V: pictures }\end{array}$} & \multirow[t]{2}{*}{$\mathrm{R}$ and $\mathrm{C}$} & $\begin{array}{l}40(60 \%) \\
\text { AVH:20 }\end{array}$ & $\begin{array}{l}41.5 \\
(9.5) \\
\end{array}$ & $\begin{array}{l}13.9 \\
(2.8) \\
\end{array}$ & $\begin{array}{l}104.0 \\
(14.7) \\
\end{array}$ & $\begin{array}{l}23.9 \\
(20.4) \\
\end{array}$ & \multirow[t]{2}{*}{ ANOVA and SNK } & \\
\hline & & & & & & & $\begin{array}{l}\text { NAVH: } \\
20\end{array}$ & $\begin{array}{l}36.9 \\
(8.9)\end{array}$ & $\begin{array}{l}14.6 \\
(3.7)\end{array}$ & $\begin{array}{l}104.3 \\
(12.6)\end{array}$ & $\begin{array}{l}15.7 \\
(20.6)\end{array}$ & & \\
\hline 2007 & Leitman [32] & $\begin{array}{c}\text { Behavioral and } \\
\text { neural (MRI): } \\
\text { Neural substrates } \\
\text { of impaired EPP }\end{array}$ & $\begin{array}{l}\text { the US } \\
\text { English }\end{array}$ & $\begin{array}{l}\text { Identification } \\
\text { (SSC) } \\
\text { Discrimination } \\
\text { (SSC pair) }\end{array}$ & A: sentences & C & $24(88 \%)$ & $\begin{array}{c}32.5 \\
(10.6)\end{array}$ & $\begin{array}{l}11.0 \\
(2.0)\end{array}$ & $94.1(7.5)$ & $\mathrm{N} / \mathrm{A}$ & $\begin{array}{c}\text { ANOVA, } \\
\text { Spearman, voxel } \\
\text { wise correlation } \\
\text { approach and PCA }\end{array}$ & \\
\hline \multirow[t]{2}{*}{2007} & \multirow[t]{2}{*}{ Shea [33] } & \multirow{2}{*}{$\begin{array}{l}\text { Behavioral: EPP } \\
\text { and auditory } \\
\text { hallucinations }\end{array}$} & \multirow[t]{2}{*}{$\begin{array}{l}\text { Australia } \\
\text { English }\end{array}$} & \multirow{2}{*}{$\begin{array}{l}\text { Identification } \\
\text { (SSC) } \\
\text { Discrimination } \\
\text { (SSC pair) }\end{array}$} & \multirow[t]{2}{*}{ A: sentences } & \multirow[t]{2}{*}{ C } & $\begin{array}{c}67(69 \%) \\
\text { AH:38 }\end{array}$ & $\begin{array}{l}40.0 \\
(10.0) \\
\end{array}$ & $\begin{array}{l}14.0 \\
(3.0) \\
\end{array}$ & $\begin{array}{l}108.0 \\
(11.0) \\
\end{array}$ & $\begin{array}{l}14.0 \\
(9.0) \\
\end{array}$ & \multirow[t]{2}{*}{$\begin{array}{l}\text { ANOVA and } \\
\text { Tukey's HSD }\end{array}$} & \\
\hline & & & & & & & $\mathrm{NAH}: 29$ & $\begin{array}{l}44.0 \\
(11.0)\end{array}$ & $14(3)$ & $\begin{array}{l}109.0 \\
(11.0)\end{array}$ & $\begin{array}{l}20.0 \\
(15.0)\end{array}$ & & \\
\hline \multirow[t]{2}{*}{2008} & \multirow[t]{2}{*}{ Chan [34] } & \multirow{2}{*}{$\begin{array}{l}\text { Behavioral: EPP } \\
\text { and } \\
\text { neuropsychological } \\
\text { function }\end{array}$} & \multirow{2}{*}{$\begin{array}{l}\text { China (HK) } \\
\text { Chinese }\end{array}$} & \multirow{2}{*}{$\begin{array}{c}\text { Identification } \\
\text { (SSC) } \\
\text { Discrimination } \\
\text { (SSC pair) } \\
\end{array}$} & \multirow{2}{*}{$\begin{array}{c}\text { A: sentences } \\
\text { (meaningless) } \\
\text { V: facial photos }\end{array}$} & \multirow[t]{2}{*}{ C } & $\begin{array}{c}43(49 \%) \\
\text { PS: } 19\end{array}$ & $\begin{array}{l}34.5 \\
(9.3) \\
\end{array}$ & $\begin{array}{l}11.7 \\
(2.1) \\
\end{array}$ & \multirow[t]{2}{*}{ N/A } & \multirow[t]{2}{*}{$\begin{array}{l}8.9 \\
(7.9)\end{array}$} & $\begin{array}{l}\text { MANOVA and } \\
\text { Multiple stepwise }\end{array}$ & \\
\hline & & & & & & & NPS:24 & $\begin{array}{l}40.0 \\
(9.1)\end{array}$ & $\begin{array}{l}8.8 \\
(3.4) \\
\end{array}$ & & & linear regression & \\
\hline 2008 & Scholten [35] & $\begin{array}{c}\text { Behavioral: } \\
\text { Gender differences }\end{array}$ & $\begin{array}{l}\text { The } \\
\text { Netherlands }\end{array}$ & $\begin{array}{l}\text { Discrimination } \\
\text { (meaning-prosody }\end{array}$ & A: sentences & C & $48(52 \%)$ & $\begin{array}{c}\text { M: } 29.5 \\
(7.0)\end{array}$ & $\mathrm{N} / \mathrm{A}$ & $\begin{array}{c}\text { M: } 107.2 \\
(9.6)\end{array}$ & $\begin{array}{c}7.0 \\
(4.9) \\
\end{array}$ & $\begin{array}{l}\text { ANOVA, } \\
\text { ANCOVA and }\end{array}$ & \\
\hline & & and IQ in EPP & Dutch & stroop test) & & & & $\begin{array}{c}\text { F: } 32.2 \\
(6.6)\end{array}$ & & $\begin{array}{c}F: 110.2 \\
(8.3)\end{array}$ & $\begin{array}{l}8.5 \\
(6.6)\end{array}$ & Pearson & \\
\hline $2009 a$ & Bach [36] & $\begin{array}{l}\text { Behavioral: High } \\
\text { clarity and EPP }\end{array}$ & $\begin{array}{l}\text { Switzerland } \\
\text { German }\end{array}$ & $\begin{array}{l}\text { Identification } \\
\quad \text { (SPP) }\end{array}$ & $\begin{array}{l}\text { A: sentences } \\
\text { (meaningless) } \\
\text { V: facial photos }\end{array}$ & C & $25(52 \%)$ & $\begin{array}{c}35.9 \\
(11.8)\end{array}$ & $\mathrm{N} / \mathrm{A}$ & N/A & $\begin{array}{c}6 \\
(\mathrm{~N} / \mathrm{A})\end{array}$ & ANOVA & \\
\hline
\end{tabular}


Table 1. Cont

\begin{tabular}{|c|c|c|c|c|c|c|c|c|c|c|c|c|c|}
\hline \multirow[b]{2}{*}{ Year } & \multirow[b]{2}{*}{ First Author } & \multirow{2}{*}{$\begin{array}{c}\text { Study Type } \\
\text { (Neurological } \\
\text { Technique): Study } \\
\text { Topic }\end{array}$} & \multirow[b]{2}{*}{$\begin{array}{c}\text { Country } \\
\text { Language }\end{array}$} & \multirow[b]{2}{*}{ Task Paradigm } & \multirow[b]{2}{*}{$\begin{array}{l}\text { Stimulus Modality: } \\
\text { Stimulus Form }\end{array}$} & \multirow[b]{2}{*}{$\begin{array}{c}\text { Response } \\
\text { Option }\end{array}$} & \multicolumn{5}{|c|}{ Patient Information } & \multirow[b]{2}{*}{$\begin{array}{l}\text { Statistical Analysis } \\
\text { Methods }\end{array}$} & \multirow[b]{2}{*}{$\begin{array}{l}\text { Notes (Reasons for } \\
\text { Exclusion from } \\
\text { Meta-Analysis) }\end{array}$} \\
\hline & & & & & & & $\begin{array}{l}\text { Number } \\
\text { (Male\%) }\end{array}$ & $\begin{array}{l}\text { Age } \\
\text { (S.D.) }\end{array}$ & $\begin{array}{l}\text { Education } \\
\text { (years) }\end{array}$ & IQ & $\begin{array}{l}\begin{array}{l}\text { Illness } \\
\text { Duration } \\
\text { (years) }\end{array} \\
\end{array}$ & & \\
\hline $2009 \mathrm{~b}$ & Bach [37] & $\begin{array}{l}\text { Behavioral and } \\
\text { neural (fMRI): } \\
\text { Lateralization of } \\
\text { EPP }\end{array}$ & $\begin{array}{c}\text { Switzerland } \\
\text { German }\end{array}$ & $\begin{array}{l}\text { Identification } \\
\quad \text { (SPP) }\end{array}$ & A: non-words & C & $15(53 \%)$ & $\begin{array}{l}31.6 \\
(7.9)\end{array}$ & N/A & $\mathrm{N} / \mathrm{A}$ & N/A & $\begin{array}{l}\text { ANOVA, ANCOVA, } \\
\text { SPM and laterality } \\
\text { measures }\end{array}$ & \\
\hline 2010 & Roux [38] & $\begin{array}{l}\text { Behavioral: } \\
\text { Implicit and } \\
\text { explicit of EPP }\end{array}$ & $\begin{array}{l}\text { France } \\
\text { French }\end{array}$ & $\begin{array}{c}\text { Identification } \\
\text { (SSC) } \\
\text { Discrimination } \\
\text { (meaning-prosody } \\
\text { stroop test) }\end{array}$ & A: words & C & $21(67 \%)$ & $\begin{array}{r}38.2 \\
(12.6)\end{array}$ & $\begin{array}{l}11.5 \\
(2.9)\end{array}$ & $\begin{array}{l}103.7 \\
(7.0)\end{array}$ & $\mathrm{N} / \mathrm{A}$ & $\begin{array}{l}\text { ANCOVA, ANOVA } \\
\text { and Pearson }\end{array}$ & \\
\hline 2012 & Gold [39] & $\begin{array}{l}\text { Behavioral: The } \\
\text { relationship } \\
\text { between auditory } \\
\text { emotion } \\
\text { recognition } \\
\text { impairments and } \\
\text { acoustic features } \\
\text { and cognition }\end{array}$ & $\begin{array}{l}\text { the US } \\
\text { English }\end{array}$ & $\begin{array}{l}\text { Identification } \\
\text { (SPP) }\end{array}$ & $\begin{array}{l}\text { A: vocal sounds (full } \\
\text { version and brief } \\
\text { version which contains } \\
\text { intensity modulation } \\
\text { and pitch modulation) } \\
\text { V: facial expressions }\end{array}$ & C & $92(86 \%)$ & $\begin{array}{l}37.8 \\
(10.4)\end{array}$ & N/A & N/A & N/A & $\begin{array}{l}\text { ANOVA, } \\
\text { multivariate } \\
\text { regression and path } \\
\text { analysis }\end{array}$ & $\begin{array}{l}\text { Identification of brie } \\
\text { version of vocal } \\
\text { sounds excluded } \\
\text { from meta-analysis } \\
\text { (task quite different } \\
\text { from other studies') }\end{array}$ \\
\hline 2013 & Ito [40] & $\begin{array}{l}\text { Behavioral: EPP } \\
\text { and positive } \\
\text { psychotic } \\
\text { symptoms }\end{array}$ & $\begin{array}{l}\text { Japan } \\
\text { Japanese }\end{array}$ & $\begin{array}{l}\text { Discrimination } \\
\text { (meaning-prosody } \\
\text { stroop test) }\end{array}$ & A: sentences & C & $28(61 \%)$ & $\begin{array}{l}30.9 \\
(8.1)\end{array}$ & $\begin{array}{l}13.6 \\
(2.0)\end{array}$ & $\mathrm{N} / \mathrm{A}$ & $\begin{array}{l}6.9 \\
(7.3)\end{array}$ & $\begin{array}{l}\text { ANOVA, } T \text { test and } \\
\text { Spearman }\end{array}$ & \\
\hline 2013 & Iwashiro [41] & $\begin{array}{l}\text { Behavioral: } \\
\text { Semantic } \\
\text { processing of } \\
\text { emotional content } \\
\text { and auditory } \\
\text { attention }\end{array}$ & $\begin{array}{l}\text { Japan } \\
\text { Japanese }\end{array}$ & $\begin{array}{l}\text { Discrimination } \\
\text { (dichotic } \\
\text { listening of SSC } \\
\text { pair) }\end{array}$ & A: words & C & $22(50 \%)$ & $\begin{array}{l}31.6 \\
(5.2)\end{array}$ & $\begin{array}{l}13.5 \\
(1.8)\end{array}$ & $\begin{array}{c}96.7 \\
(10.0)\end{array}$ & $\begin{array}{l}8.7 \\
(6.7)\end{array}$ & $\begin{array}{c}\text { ANOVA, } \\
\text { Paired-samples } T \text { test } \\
\text { and Spearman }\end{array}$ & \\
\hline 2013 & Jahshan [42] & $\begin{array}{l}\text { Behavioral and } \\
\text { neural study } \\
\text { (ERP): Auditory } \\
\text { processing and } \\
\text { EPP }\end{array}$ & $\begin{array}{l}\text { the US } \\
\text { English }\end{array}$ & $\begin{array}{l}\text { Identification } \\
\text { (SSC) }\end{array}$ & $\begin{array}{l}\text { A: sentences V: photos } \\
\text { of facial expressions }\end{array}$ & C & $36(69 \%)$ & $\begin{array}{l}47.7 \\
(10.0)\end{array}$ & $\begin{array}{l}12.6 \\
(1.8)\end{array}$ & $\mathrm{N} / \mathrm{A}$ & $\begin{array}{c}24.3 \\
(11.5)\end{array}$ & $\begin{array}{l}\text { Independent-samples } \\
T \text { test, Chi-square } \\
\text { test, One-sample } T \\
\text { test, MANOVA, } \\
\text { Pearson and } \\
\text { Multiple regression } \\
\text { analysis } \\
\end{array}$ & \\
\hline 2013 & $\begin{array}{c}\text { Kantrow-itz } \\
{[43]}\end{array}$ & $\begin{array}{c}\text { Behavioral: } \\
\text { Emotion } \\
\text { recognition based } \\
\text { on tone of voice } \\
\text { and basic auditory } \\
\text { processing }\end{array}$ & $\begin{array}{l}\text { the US } \\
\text { English }\end{array}$ & $\begin{array}{l}\text { Identification } \\
\text { (SSC and SPP) }\end{array}$ & $\begin{array}{l}\text { A: FM tones and } \\
\text { sentences }\end{array}$ & $\mathrm{C}$ and $\mathrm{R}$ & $41(53 \%)$ & $\begin{array}{l}36.5 \\
(10.9)\end{array}$ & $\begin{array}{l}12.3 \\
(2.3)\end{array}$ & $\mathrm{N} / \mathrm{A}$ & $\begin{array}{c}16.0 \\
(10.0)\end{array}$ & $\begin{array}{c}\text { ANOVA, } \\
\text { Independent-samples } \\
T \text { test, } \mathrm{M}-\mathrm{W} \text { test, } \\
\text { multivariate linear } \\
\text { regression and } \\
\text { Pearson }\end{array}$ & \\
\hline \multirow[t]{2}{*}{2013} & \multirow[t]{2}{*}{$\begin{array}{l}\text { McLach-lan } \\
\text { [44] }\end{array}$} & \multirow{2}{*}{$\begin{array}{l}\text { Behavioral: EPP } \\
\text { and auditory } \\
\text { hallucinations }\end{array}$} & \multirow[t]{2}{*}{$\begin{array}{l}\text { Australia } \\
\text { English }\end{array}$} & \multirow[t]{2}{*}{$\begin{array}{l}\text { Identification } \\
\quad \text { (SSC) }\end{array}$} & \multirow[t]{2}{*}{ A: sentences } & \multirow[t]{2}{*}{$\mathrm{R}$} & $\begin{array}{l}34(76 \%) \\
\text { AVH:19 }\end{array}$ & $\begin{array}{l}41.2 \\
(9.9) \\
\end{array}$ & $\begin{array}{l}13.2 \\
(2.5) \\
\end{array}$ & $\begin{array}{l}104.2 \\
(8.8)\end{array}$ & \multirow[t]{2}{*}{ N/A } & \multirow{2}{*}{$\begin{array}{c}\text { ANOVA, Chi-square } \\
\text { test and } \\
\text { Independent-samples } \\
T \text { test }\end{array}$} & \\
\hline & & & & & & & NAVH:15 & $\begin{array}{l}43.5 \\
(8.8)\end{array}$ & $\begin{array}{l}12.9 \\
(2.6)\end{array}$ & $\begin{array}{c}101.2 \\
(9.7)\end{array}$ & & & \\
\hline
\end{tabular}


Table 1. Cont

\begin{tabular}{|c|c|c|c|c|c|c|c|c|c|c|c|c|c|}
\hline \multirow[b]{2}{*}{ Year } & \multirow[b]{2}{*}{ First Author } & \multirow{2}{*}{$\begin{array}{c}\text { Study Type } \\
\text { (Neurological } \\
\text { Technique: Study } \\
\text { Topic }\end{array}$} & \multirow[b]{2}{*}{$\begin{array}{c}\text { Country } \\
\text { Language }\end{array}$} & \multirow[b]{2}{*}{ Task Paradigm } & \multirow[b]{2}{*}{$\begin{array}{l}\text { Stimulus Modality: } \\
\text { Stimulus Form }\end{array}$} & \multirow[b]{2}{*}{$\begin{array}{c}\text { Response } \\
\text { Option }\end{array}$} & \multicolumn{5}{|c|}{ Patient Information } & \multirow[b]{2}{*}{$\begin{array}{c}\text { Statistical } \\
\text { Analysis Methods }\end{array}$} & \multirow[b]{2}{*}{$\begin{array}{l}\text { Notes (Reasons for } \\
\text { Exclusion from } \\
\text { Meta-Analysis) }\end{array}$} \\
\hline & & & & & & & $\begin{array}{l}\text { Number } \\
\text { (Male\%) }\end{array}$ & $\begin{array}{l}\text { Age } \\
\text { (S.D.) }\end{array}$ & $\begin{array}{l}\text { Education } \\
\text { (years) }\end{array}$ & IQ & $\begin{array}{l}\begin{array}{l}\text { Illness } \\
\text { Duration } \\
\text { (years) }\end{array} \\
\end{array}$ & & \\
\hline 2013 & Pinheiro [45] & $\begin{array}{c}\text { Behavioral and } \\
\text { neural (ERP): ERP } \\
\text { correlates of EPP }\end{array}$ & $\begin{array}{l}\text { the US } \\
\text { English }\end{array}$ & $\begin{array}{l}\text { Identification } \\
\text { (SSC and SPP) }\end{array}$ & $\begin{array}{l}\text { A: sentences and } \\
\text { sentences } \\
\text { (meaningless) }\end{array}$ & C & $15(100 \%)$ & $\begin{array}{l}49.7 \\
(9.1)\end{array}$ & $\begin{array}{l}14.33 \\
(1.8)\end{array}$ & $\begin{array}{r}92.2 \\
(29.4)\end{array}$ & $\begin{array}{l}22.3 \\
(10.5)\end{array}$ & $\begin{array}{l}\text { MANOVA and } \\
\text { Spearman }\end{array}$ & \\
\hline 2013 & Tseng [46] & $\begin{array}{l}\text { Behavioral: EPP } \\
\text { across modalities } \\
\text { and psychotic } \\
\text { symptoms }\end{array}$ & $\begin{array}{l}\text { China } \\
\text { (Taiwan) } \\
\text { Chinese }\end{array}$ & $\begin{array}{l}\text { Identification } \\
\quad \text { (SPP) }\end{array}$ & $\begin{array}{l}\text { A: sounds } \\
\text { V: facial photos }\end{array}$ & C & $111(46 \%)$ & $\begin{array}{c}38.2 \\
(10.1)\end{array}$ & $\begin{array}{l}15.9 \\
(3.4)\end{array}$ & $\begin{array}{c}92.5 \\
(16.3)\end{array}$ & $\begin{array}{l}13.8 \\
(9.7)\end{array}$ & $\begin{array}{l}\text { Independent-samples } \\
T \text { test, Chi-square } \\
\text { test, ANCOVA, } \\
\text { Pearson, Multiple } \\
\text { bidirectional } \\
\text { stepwise linear } \\
\text { regression, and } \\
\text { Multiple linear } \\
\text { regression }\end{array}$ & \\
\hline 2014 & Brazo [47] & $\begin{array}{l}\text { Behavioral: EP } \\
\text { comprehension } \\
\text { and semantic } \\
\text { content }\end{array}$ & $\begin{array}{l}\text { France } \\
\text { French }\end{array}$ & $\begin{array}{c}\text { Semantic } \\
\text { identification } \\
\text { (EPP influences) }\end{array}$ & A: sentences & C & $16(56 \%)$ & $\begin{array}{l}39.7 \\
(8.6)\end{array}$ & $\mathrm{N} / \mathrm{A}$ & $\begin{array}{c}89.8 \\
(11.5)\end{array}$ & $\begin{array}{l}13.3 \\
(5.8)\end{array}$ & $\begin{array}{c}\text { ANOVA, } \\
\text { ANCOVA and } \\
\text { Paired-samples } T \\
\text { test }\end{array}$ & \\
\hline 2014 & $\begin{array}{l}\text { Dondai-ne } \\
{[48]}\end{array}$ & $\begin{array}{l}\text { Behavioral: facial } \\
\text { and vocal emotion } \\
\text { recognition biases }\end{array}$ & $\begin{array}{l}\text { France } \\
\text { French }\end{array}$ & $\begin{array}{c}\text { Emotion } \\
\text { intensity rating }\end{array}$ & $\begin{array}{l}\text { A: non-verbal bursts } \\
\text { without semantic } \\
\text { content (vowel "ah") } \\
\text { V: facial expressions }\end{array}$ & $\mathrm{R}$ & $23(65 \%)$ & $\begin{array}{l}33.9 \\
(7.3)\end{array}$ & $\begin{array}{l}12.7 \\
(2.0)\end{array}$ & $\mathrm{N} / \mathrm{A}$ & $\begin{array}{l}12.4 \\
(6.5)\end{array}$ & $\begin{array}{c}\text { ANOVA, } \\
\text { independent } \\
\text { sample } T \text { test, } \\
\text { Spearman's } \\
\text { correlation } \\
\text { analysis }\end{array}$ & $\begin{array}{l}\text { The whole study } \\
\text { excluded from } \\
\text { meta-analysis (task } \\
\text { quite different from } \\
\text { other studies') }\end{array}$ \\
\hline 2014 & $\begin{array}{c}\text { Hoertna-gl } \\
\text { [11] }\end{array}$ & $\begin{array}{c}\text { Behavioral: A } \\
\text { comparison of EPP } \\
\text { between } \\
\text { symptomatically } \\
\text { remitted patients } \\
\text { with schizophrenia } \\
\text { and bipolar } \\
\text { disorder }\end{array}$ & $\begin{array}{l}\text { Austria } \\
\text { N/A }\end{array}$ & $\begin{array}{l}\text { Identification } \\
\text { (SSC) }\end{array}$ & A: sentences & C & $41(54 \%)$ & $\begin{array}{l}40.5 \\
(8.5)\end{array}$ & $\begin{array}{l}12.9 \\
(2.9)\end{array}$ & $\mathrm{N} / \mathrm{A}$ & $\begin{array}{l}12.4 \\
(6.9)\end{array}$ & $\begin{array}{l}\text { Chi-square test, } \\
\text { ANOVA, multiple } \\
\text { linear regression }\end{array}$ & \\
\hline 2014a & $\begin{array}{c}\text { Kantrow-itz } \\
\text { [19] }\end{array}$ & $\begin{array}{l}\text { Behavioral and } \\
\text { neural (MRI): early } \\
\text { sensory processing } \\
\text { and sarcasm } \\
\text { perception }\end{array}$ & $\begin{array}{l}\text { the US } \\
\text { English }\end{array}$ & $\begin{array}{c}\text { Identification } \\
\text { (SSC) } \\
\text { Discrimination } \\
\text { (sarcasm } \\
\text { perception) }\end{array}$ & A: sentences & C & $76(63 \%)$ & $\begin{array}{c}37.4 \\
(10.1)\end{array}$ & $\begin{array}{c}12.1 \\
(2.3) \\
(74 \\
\text { patients) }\end{array}$ & $\mathrm{N} / \mathrm{A}$ & $\begin{array}{c}15.3 \\
(9.0) \\
(74 \\
\text { patients) }\end{array}$ & $\begin{array}{c}\text { ANOVA, } \\
\text { independent } \\
\text { sample } T \text { test, } \\
\text { Pearson } \\
\text { correlations and } \\
\text { multivariate linear } \\
\text { regression }\end{array}$ & $\begin{array}{l}\text { Discrimination task } \\
\text { excluded from } \\
\text { meta-analysis } \\
\text { (sarcasm is a } \\
\text { complex social } \\
\text { emotion) }\end{array}$ \\
\hline $2014 \mathrm{~b}$ & $\begin{array}{c}\text { Kantrow-itz } \\
{[49]}\end{array}$ & $\begin{array}{l}\text { Behavioral: } \\
\text { amusia and } \\
\text { protolanguage } \\
\text { impairments in } \\
\text { schizophrenia }\end{array}$ & $\begin{array}{l}\text { the US } \\
\text { English }\end{array}$ & $\begin{array}{l}\text { Identification } \\
\text { (emotional } \\
\text { categories and } \\
\text { intensity of SSC) }\end{array}$ & A: phrases & $\mathrm{R}$ & $31(87 \%)$ & $\begin{array}{l}39.4 \\
(9.9)\end{array}$ & $\begin{array}{c}11.4 \\
(2.2) \\
(29 \\
\text { patients })\end{array}$ & $\mathrm{N} / \mathrm{A}$ & $\begin{array}{c}14.8 \\
(8.2) \\
(24 \\
\text { patients) }\end{array}$ & $\begin{array}{c}\text { independent-samples } \\
T \text { test, MANOVA, } \\
\text { ANOVA, } \\
\text { multivariate linear } \\
\text { regression and } \\
\text { Pearson }\end{array}$ & $\begin{array}{l}\text { Identification of } \\
\text { emotional intensity } \\
\text { excluded from } \\
\text { meta-analysis (tasks } \\
\text { quite different from } \\
\text { other studies') }\end{array}$ \\
\hline
\end{tabular}


Table 1. Cont

\begin{tabular}{|c|c|c|c|c|c|c|c|c|c|c|c|c|c|}
\hline \multirow[b]{2}{*}{ Year } & \multirow[b]{2}{*}{ First Author } & \multirow{2}{*}{$\begin{array}{c}\text { Study Type } \\
\text { (Neurological } \\
\text { Technique): Study } \\
\text { Topic }\end{array}$} & \multirow[b]{2}{*}{$\begin{array}{c}\text { Country } \\
\text { Language }\end{array}$} & \multirow[b]{2}{*}{ Task Paradigm } & \multirow[b]{2}{*}{$\begin{array}{l}\text { Stimulus Modality: } \\
\text { Stimulus Form }\end{array}$} & \multirow[b]{2}{*}{$\begin{array}{c}\text { Response } \\
\text { Option }\end{array}$} & \multicolumn{5}{|c|}{ Patient Information } & \multirow[b]{2}{*}{$\begin{array}{c}\text { Statistical } \\
\text { Analysis Methods }\end{array}$} & \multirow[b]{2}{*}{$\begin{array}{l}\text { Notes (Reasons for } \\
\text { Exclusion from } \\
\text { Meta-Analysis) }\end{array}$} \\
\hline & & & & & & & $\begin{array}{l}\text { Number } \\
\text { (Male\%) }\end{array}$ & $\begin{array}{l}\text { Age } \\
\text { (S.D.) }\end{array}$ & $\begin{array}{l}\text { Education } \\
\text { (years) }\end{array}$ & IQ & $\begin{array}{l}\text { Illness } \\
\text { Duration } \\
\text { (years) }\end{array}$ & & \\
\hline 2014 & Müller [50] & $\begin{array}{l}\text { Behavioral and } \\
\text { neural study } \\
\text { (ERP): Neural } \\
\text { substrates of } \\
\text { auditory emotion } \\
\text { recognition deficits }\end{array}$ & $\begin{array}{l}\text { the US } \\
\text { English }\end{array}$ & $\begin{array}{l}\text { Discrimination } \\
\text { (face-prosody } \\
\text { stroop test) }\end{array}$ & $\begin{array}{l}\text { A and V: sounds and } \\
\text { pictures of faces }\end{array}$ & $\mathrm{R}$ & $15(73 \%)$ & $\begin{array}{l}35.1 \\
(9.3)\end{array}$ & $\begin{array}{l}14.1 \\
(2.2)\end{array}$ & N/A & $\begin{array}{l}14.3 \\
(9.1)\end{array}$ & $\begin{array}{l}\text { ANOVA, } \\
\text { MANOVA, } \\
\text { Bonferroni } \\
\text { correction and } \\
\text { Pearson }\end{array}$ & \\
\hline 2014 & Pinheiro [51] & $\begin{array}{l}\text { Behavioral and } \\
\text { neural study } \\
\text { (ERP): ERP } \\
\text { correlates of EPP }\end{array}$ & $\begin{array}{l}\text { the US } \\
\text { English }\end{array}$ & $\begin{array}{l}\text { Identification } \\
\text { (SSC and SPP) }\end{array}$ & $\begin{array}{l}\text { A: words and } \\
\text { non-words }\end{array}$ & C & $16(69 \%)$ & $\begin{array}{l}48.9 \\
(7.4)\end{array}$ & $\begin{array}{l}14.0 \\
(2.4)\end{array}$ & $>85$ & $\begin{array}{l}19.5 \\
(11.0)\end{array}$ & ANOVA & \\
\hline 2015 & $\begin{array}{l}\text { Corcora-n } \\
{[52]}\end{array}$ & $\begin{array}{c}\text { Behavioral: } \\
\text { emotion } \\
\text { recognition deficits } \\
\text { as predictors of } \\
\text { transition in } \\
\text { individual at } \\
\text { clinical high risk } \\
\text { for schizophrenia } \\
\end{array}$ & $\begin{array}{l}\text { the US } \\
\text { English }\end{array}$ & $\begin{array}{l}\text { Identification } \\
\quad \text { (SSC) }\end{array}$ & $\begin{array}{c}\text { A: sentences } \\
\text { V: facial expressions }\end{array}$ & C & $7(57 \%)$ & $\begin{array}{l}20.0 \\
(5.2)\end{array}$ & N/A & N/A & N/A & ANOVA & $\begin{array}{l}\text { The whole study } \\
\text { excluded from } \\
\text { meta-analysis } \\
\text { (involving } \\
\text { prodromal patients) }\end{array}$ \\
\hline 2015 & $\begin{array}{c}\text { Kantrow-itz } \\
\text { [53] }\end{array}$ & $\begin{array}{l}\text { Behavioral and } \\
\text { neural study (ERP } \\
\text { and rsfMRI): } \\
\text { Neural substrates } \\
\text { of auditory } \\
\text { emotion } \\
\text { recognition deficits }\end{array}$ & $\begin{array}{l}\text { the US } \\
\text { English }\end{array}$ & $\begin{array}{l}\text { Identification } \\
\text { (SSC and SPP) }\end{array}$ & $\begin{array}{l}\text { A: FM tones and } \\
\text { sentences }\end{array}$ & C & $84(81 \%)$ & $\begin{array}{l}39.4 \\
(10.6)\end{array}$ & $\mathrm{N} / \mathrm{A}$ & N/A & $\begin{array}{l}15.9 \\
(9.4)\end{array}$ & $\begin{array}{l}\text { ANOVA, } \\
\text { MANOVA, } \\
\text { Independent-samples } \\
T \text { test, multivariate } \\
\text { linear regression, } \\
\text { Pearson and SPM }\end{array}$ & \\
\hline 2015 & $\begin{array}{c}\text { Regenbo-gen } \\
{[18]}\end{array}$ & $\begin{array}{l}\text { Behavioral and } \\
\text { neural (fMRI): } \\
\text { Neural responses } \\
\text { to multimodal } \\
\text { stimuli and } \\
\text { pathology-specific } \\
\text { social cognition } \\
\text { deficits } \\
\end{array}$ & $\begin{array}{l}\text { Germany } \\
\text { German }\end{array}$ & Empathy rating & $\begin{array}{l}\text { A and V: video clips } \\
\text { expressing emotion } \\
\text { through three channels: } \\
\text { facial expression, } \\
\text { prosody and content }\end{array}$ & $\mathrm{R}$ & 20 (N/A) & $\begin{array}{l}37.3 \\
(8.4)\end{array}$ & N/A & N/A & N/A & $\begin{array}{c}\text { ANOVA, Pearson } \\
\text { correlation } \\
\text { analysis and SPM }\end{array}$ & $\begin{array}{l}\text { The whole study } \\
\text { excluded from } \\
\text { meta-analysis } \\
\text { (involving } \\
\text { participants' } \\
\text { empathy, which is a } \\
\text { complex social } \\
\text { emotion) }\end{array}$ \\
\hline 2015 & Sterea [9] & $\begin{array}{l}\text { Behavioral: the } \\
\text { relationship } \\
\text { between social } \\
\text { cognition and } \\
\text { functional } \\
\text { outcomes in } \\
\text { schizophrenia }\end{array}$ & $\begin{array}{l}\text { Romania } \\
\text { Romanian }\end{array}$ & $\begin{array}{l}\text { Definition of } \\
\text { emotion and } \\
\text { explanation of } \\
\text { emotional } \\
\text { situations and } \\
\text { events }\end{array}$ & N/A & interview & $15(60 \%)$ & $\begin{array}{l}41.9 \\
(8.4)\end{array}$ & $\mathrm{N} / \mathrm{A}$ & N/A & $\mathrm{N} / \mathrm{A}$ & $\begin{array}{l}\text { M-U test and } \\
\text { Kendall } \\
\text { correlation }\end{array}$ & $\begin{array}{l}\text { The whole study } \\
\text { excluded from } \\
\text { meta-analysis (task } \\
\text { quite different from } \\
\text { other studies' and } \\
\text { involving complex } \\
\text { emotions such as } \\
\text { surprise and } \\
\text { suspiciousness) }\end{array}$ \\
\hline 2015 & $\begin{array}{l}\text { Weisger-ber } \\
\text { [54] }\end{array}$ & $\begin{array}{l}\text { Behavioral: Facial, } \\
\text { vocal and musical } \\
\text { emotion } \\
\text { recognition }\end{array}$ & $\begin{array}{l}\text { Belgium } \\
\text { French }\end{array}$ & $\begin{array}{l}\text { Identification } \\
\quad \text { (SPP) }\end{array}$ & $\begin{array}{c}\text { A: non-verbal vocal } \\
\text { affect bursts } \\
\text { V: computer-generated } \\
\text { faces }\end{array}$ & $\mathrm{R}$ & $30(37 \%)$ & $\begin{array}{l}35.5 \\
(12.7)\end{array}$ & $12(2.2)$ & N/A & $\begin{array}{l}10.9 \\
(9.4)\end{array}$ & $\begin{array}{l}\text { ANOVA, } \\
\text { MANOVA and } \\
\text { Spearman }\end{array}$ & \\
\hline
\end{tabular}


Table 1. Cont.

\begin{tabular}{|c|c|c|c|c|c|c|c|c|c|c|c|c|c|}
\hline \multirow[b]{2}{*}{ Year } & \multirow[b]{2}{*}{ First Author } & \multirow{2}{*}{$\begin{array}{c}\text { Study Type } \\
\text { (Neurological } \\
\text { Technique): Study } \\
\text { Topic }\end{array}$} & \multirow[b]{2}{*}{$\begin{array}{c}\text { Country } \\
\text { Language }\end{array}$} & \multirow[b]{2}{*}{ Task Paradigm } & \multirow[b]{2}{*}{$\begin{array}{l}\text { Stimulus Modality: } \\
\text { Stimulus Form }\end{array}$} & \multirow[b]{2}{*}{$\begin{array}{l}\text { Response } \\
\text { Option }\end{array}$} & \multicolumn{5}{|c|}{ Patient Information } & \multirow[b]{2}{*}{$\begin{array}{c}\text { Statistical } \\
\text { Analysis Methods }\end{array}$} & \multirow[b]{2}{*}{$\begin{array}{l}\text { Notes (Reasons for } \\
\text { Exclusion from } \\
\text { Meta-Analysis) }\end{array}$} \\
\hline & & & & & & & $\begin{array}{l}\text { Number } \\
(\text { Male\%) }\end{array}$ & $\begin{array}{l}\text { Age } \\
\text { (S.D.) }\end{array}$ & $\begin{array}{l}\text { Education } \\
\text { (years) }\end{array}$ & IQ & $\begin{array}{l}\begin{array}{l}\text { Illness } \\
\text { Duration } \\
\text { (years) }\end{array} \\
\end{array}$ & & \\
\hline 2016 & $\begin{array}{c}\text { Razafim-andimby } \\
{[55]}\end{array}$ & $\begin{array}{c}\text { Behavioral and } \\
\text { neuraral study } \\
\text { (fMRI): Neural } \\
\text { bases of emotional } \\
\text { sentence } \\
\text { attribution }\end{array}$ & $\begin{array}{l}\text { France } \\
\text { French }\end{array}$ & $\begin{array}{l}\text { Identification } \\
\quad \text { (SSC) }\end{array}$ & A: sentences & C & $21(76 \%)$ & $\begin{array}{l}33.9 \\
(7.4)\end{array}$ & N/A & $\mathrm{N} / \mathrm{A}$ & $\begin{array}{l}11.9 \\
(7.9)\end{array}$ & $\begin{array}{l}\text { MANOVA, } \\
\text { Chi-square test, } \\
\text { SPM and Wilcoxon } \\
\text { Rank Test }\end{array}$ & \\
\hline 2017 & Hernim-an [56] & $\begin{array}{l}\text { Secondary } \\
\text { analysis: the effect } \\
\text { of comorbid } \\
\text { depression on } \\
\text { facial and prosody } \\
\text { emotion } \\
\text { recognition }\end{array}$ & $\begin{array}{l}\text { Australia } \\
\text { English }\end{array}$ & N/A & N/A & N/A & $\begin{array}{c}82 \\
(65.9 \%)\end{array}$ & $\begin{array}{l}21.1 \\
(2.6)\end{array}$ & $\mathrm{N} / \mathrm{A}$ & $\begin{array}{l}93.3 \\
(13.2)\end{array}$ & N/A & $\begin{array}{l}\text { ANCOVA and } \\
\text { partial correlation } \\
\text { analysis }\end{array}$ & $\begin{array}{l}\text { The whole study } \\
\text { excluded from } \\
\text { meta-analysis } \\
\text { (involving } \\
\text { participants with } \\
\text { comorbid disorders } \\
\text { and secondary } \\
\text { analysis of the data) }\end{array}$ \\
\hline 2018 & Pawełcz-yk [57] & $\begin{array}{l}\text { Behavioral: } \\
\text { Extralinguistic and } \\
\text { paralinguistic } \\
\text { processing } \\
\text { meditated by right } \\
\text { hemisphere }\end{array}$ & $\begin{array}{l}\text { Poland } \\
\text { Polish }\end{array}$ & $\begin{array}{l}\text { Identification } \\
\quad \text { (SPP) }\end{array}$ & $\begin{array}{l}\text { A: sentences } \\
\text { (meaningless) }\end{array}$ & C & $40(58 \%)$ & $\begin{array}{l}26.3 \\
(9.3)\end{array}$ & $\begin{array}{l}12.0 \\
(2.6)\end{array}$ & N/A & $\begin{array}{r}3.9 \\
(4.7)\end{array}$ & $\begin{array}{l}T \text { test, Chi-square } \\
\text { Test and M-U test }\end{array}$ & \\
\hline & $\begin{array}{l}\text { (1) Abbreviat } \\
\text { semantic conte } \\
\text { ory and visual } \\
\text { ysis of Varianc } \\
\text { ipal Compone } \\
\text { es regardless o }\end{array}$ & $\begin{array}{l}\text { ns: fMRI = func } \\
\text {; SPP = stimuli } \\
\text { imuli simultanec } \\
\text { ANCOVA = An } \\
\text { Analysis; SPM } \\
\text { lifferent task pa }\end{array}$ & $\begin{array}{l}\text { isped } \\
\text { is of Cc } \\
\text { atistica } \\
\text { gms. (3 }\end{array}$ & $\begin{array}{l}\text { resonance in } \\
\text { dy (without } \\
\text { in the same to } \\
\text { ance; MANO } \\
\text { ametric Map } \\
42.5(7.2) \text {, the }\end{array}$ & $\begin{array}{l}\text { g; ERP = even } \\
\text { ntic content); } \mathrm{F} \\
\text { VH = auditory } \\
=\text { Multivariate } \\
\mathrm{N} / \mathrm{A}=\text { not av } \\
\text { in is } 42.5 \text { and S. }\end{array}$ & $\begin{array}{l}\text { ted pot } \\
\text { ne = fre } \\
1 \text { halluc } \\
\text { sis of V } \\
\text { e. (2) } P \\
7.2 \text {. }\end{array}$ & $\begin{array}{l}\text { ial; rsfM } \\
\text { ency-mo } \\
\text { ation; N } \\
\text { lance; M } \\
\text { ents' info }\end{array}$ & $\begin{array}{l}=\text { Mar } \\
\text { nation }\end{array}$ & $\begin{array}{l}\text { state fu } \\
\text {; } \mathrm{C}=\mathrm{ch} \\
\text { uditory } \\
\text { Whitney } \\
\text { olves al }\end{array}$ & $\begin{array}{l}\text { Irbal } \\
\text { J test } \\
\text { he p }\end{array}$ & $\begin{array}{l}\text { gnetic re } \\
\text { ting; A = } \\
\text { cination; } \\
\text { K = Stud } \\
\text { ts who p }\end{array}$ & $\begin{array}{l}\text { esonance imaging } \\
\text { auditory; } \mathrm{V}=\text { vi } \\
\mathrm{M}=\text { male; } \mathrm{F}=\mathrm{fen} \\
\text { ent-Newman-Ke } \\
\text { articipated in the }\end{array}$ & $\begin{array}{l}\text { SSC }=\text { stimuli } \\
\text { al; } \text { A and V = } \\
\text { le; ANOVA = } \\
\text { ls test; PCA = } \\
\text { orresponding }\end{array}$ \\
\hline
\end{tabular}




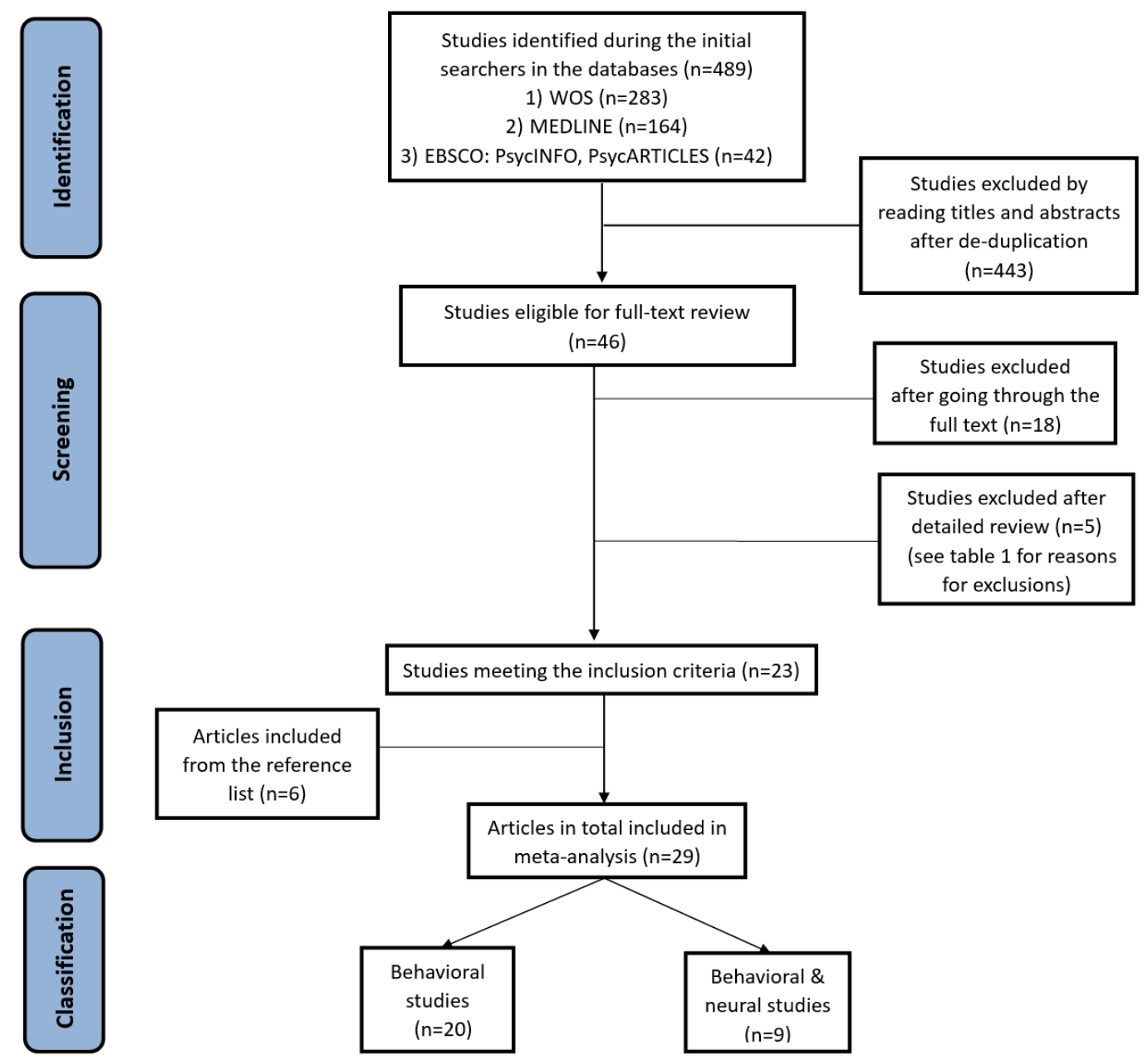

Figure 1. Flowchart of selecting studies for review.

\subsubsection{Data Synthesis}

A narrative synthesis method was employed to describe and compare the selected studies by summarizing their research topics, task paradigms, stimulus presentation, response options, patients' backgrounds, statistical analysis methods, participants' overall and single emotion processing performance. A quantitative meta-analytic approach was adopted to calculate the effect sizes (Cohen's d) for the difference in EPP between schizophrenic patients and healthy controls based on the reported statistics. Cohen's d was calculated with an online meta-analysis effect size calculator developed by David Wilson [58]. Means and standard deviations, if reported in the selected research, were used for calculation of individual studies. Otherwise, reported $t$ or $F$ statistics were used. The values of effect size were calculated in terms of two experimental paradigms: identification and discrimination test. For the studies that applied more than one task measuring EPP of patients and healthy controls or more than one subtype of schizophrenic patients in each paradigm [31,33], the performance results in these tasks or patient groups were averaged and taken together as one value.

The computed effect sizes of 29 individual studies were then further analyzed using Comprehensive Meta-Analysis Version 2.0 [59]. Studies were weighted in order to control the differences in sample size. The mean effect sizes across all studies (including both identification and discrimination tasks) were calculated and presented in a forest plot using the random-effects model since the selected studies differed from one another in study populations which could influence the treatment effect [60]. We categorized the cutoff points of effect size as small $(d=0.2)$, medium $(d=0.5)$ or large $(d=0.8)$ [61]. Publication bias was also assessed graphically using a funnel plot. 


\section{Results}

\subsection{General Characteristics of the Included Studies}

Table 1 summarizes the general characteristics of the related studies, including study types, topics, task paradigms, stimulus modalities and forms, response options, study participants, patients' demographic and clinical information, statistical analysis methods and reasons for exclusion from meta-analysis.

\subsubsection{Study Topics}

Out of the 29 studies selected for review, all employed behavioral tasks, and only 9 included neurophysiological data. While all the 29 studies examined the perception of emotional prosody in schizophrenic patients, only 2 involved the production of EP [28,31]. Therefore, we will mainly discuss the emotional prosody perception in this review.

One central topic of the behavioral studies was the relationship between EPP and its influential factors. Non-linguistic influential factors of EPP such as emotion clarity, gender, IQ, types and models of processing, psychotic symptoms, hemispheric dysfunctions and neuropsychological function had been focal points of attention [28,34-36,38,40,46,54,57]. In recent years, a few studies also examined linguistic factors influencing patients' performance such as tone of voice [43] and semantic contents [47]. Another main topic was the relationship between emotional prosody and other cognitive and social indices such as social functioning [29], auditory processing [30,41] and auditory hallucinations $[31,33,44]$.

Among the selected cognitive neuroscience studies, magnetic resonance imaging (MRI) and functional magnetic resonance imaging (fMRI) were employed to study the hemispheric processing and spatial localization of EPP $[32,37,53,55]$. Event-related potential (ERP) correlates were also under the exploration of several studies to examine the temporal processing during emotional prosody perception $[42,45,50,51,53]$.

\subsubsection{Task Paradigms, Stimulus Presentation and Response Options}

The test paradigms of EPP in the selected studies generally fell into two major categories: identification task (24 studies) and discrimination task (9 studies) with some studies using both tasks. In the identification tasks (also called explicit EPP task), participants were usually instructed to recognize the emotion that the voice expressed in a semantically neutral sentence [62]. To measure EPP on a more implicit level, discrimination tasks were often used, in which participants were asked to differentiate either the stimuli presented in pairs (5 studies) or judge congruency between spoken verbal material with either congruent or incongruent emotional valences (4 studies). The former tasks were usually combined with a dichotic listening paradigm where participants were asked to attentively focus on one of the stimuli when they were presented simultaneously to both ears. The latter case was analogous to Stroop tests, and the participants were instructed to focus on either prosodic congruency or semantic congruency in separate test trials.

Verbal stimuli with semantic content (SSC) and stimuli that kept the emotional prosody information without semantic content (SPP) were employed in the selected studies. SSC varied in different linguistic units, covering syllables, words, phrases and sentences. SPP were also presented in various forms such as vocal sounds, frequency-modulated (FM) tones, asyllabic sounds, non-words and meaningless sentences. All studies adopted auditory stimuli and a few also tested facial emotion recognition in their studies. However, only one study combined visual stimuli (pictures) and auditory stimuli (sounds) simultaneously in the same task [50].

\subsubsection{Study Participants}

The studies were conducted in a number of countries, including the United States (13), Australia (3), France (3), Japan (2), China (2), Switzerland (2), Belgium (1), The Netherlands (1), 
Austria (1), and Poland (1). Ethnicities of participants in each country were not explicitly indicated in all studies. English (16) was the major language used in the selected studies, followed by French (2), German (2), Japanese (2), Mandarin Chinese (2), Polish (1), and Dutch (1), whereas one study did not specify the task language. Interestingly, studies in non-tonal languages (e.g., English, German, Dutch, Japanese and Polish) significantly outnumbered tonal languages (e.g., Mandarin Chinese), and many more studies were conducted in stress-timed languages (e.g., English, German, Polish and Dutch) than syllable-timed languages (e.g., French and Mandarin Chinese). All studies chose healthy participants as control. There was one study which not only used healthy controls, but also selected patients with left brain damage (LBD) and right brain damage (RBD) for comparison of patient profiles in affective-prosodic deficits [28]. In two other studies, patients with depression and bipolar disorder were also included as a comparison group in addition to healthy controls [11,36].

As described in the selected articles, different types of schizophrenia or schizoaffective disorders were involved, including chronic schizophrenia (CS), paranoid schizophrenia (PS), schizophrenia with auditory verbal hallucination $(\mathrm{SAVH})$, a disorganized type, and a residual subtype. There were five subtypes of schizophrenia in the fourth edition of the Diagnostic and Statistical Manual of Mental Disorders (DSM-IV), respectively known as paranoid, disorganized, catatonic, undifferentiated, and residual. For instance, disorganized schizophrenia is characterized by disorganized symptoms including speech, behavior, and flat or inappropriate affect, whereas residual schizophrenia refers to much alleviated cases in which the patient no longer shows the prominent schizophrenic symptoms but some hallucinations or idiosyncratic behaviors may still be present. One important note here is that the selected studies in our review cover the period of 2001 to 2018. Within this time frame, there have been drastic changes from the fourth edition to the current fifth edition (DSM-V). In DSM-V, however, the five subtypes of schizophrenia are no longer included as the American Psychiatric Association determined that these distinctions were not helpful to clinicians because patients' symptoms may overlap and change from one subtype to another.

\subsubsection{Reporting of Key Demographic and Clinical Information of Schizophrenic Patients}

Table 1 also illustrates the reporting of some basic information of the schizophrenic patients. The data presented this table involve patients who participated in the corresponding studies regardless of different tasks paradigms. The sample sizes vary tremendously with the number of schizophrenic patients ranging from 15 to 111 with the mean standing at 39.3 and standard deviation at 24.3, and the average percentage of male was $64.0 \%$. Age was reported by all studies and more than half of the studies included information such as mean years of education and IQ. Handedness, participants' socioeconomic status, parents' socioeconomic status, race, parental education, musical background and employment were additional information only recorded by several studies $[41,51,53]$.

The included studies reported duration of illness (20) twice more frequently than age of onset (8). The Positive and Negative Syndrome Scale (PANSS) (18), the Scale for the Assessment of Positive Symptoms (SAPS) (8), the Scale for the Assessment of Negative Symptoms (SANS) (11) and the Brief Psychiatric Rating Scale (BPRS) (5) were widely-acknowledged as systematic protocols for studies to retrieve symptomatic data, but none of them were administered by more than $60 \%$ of the selected studies. Twenty-three studies contained medical information with either the type or dose of medication specified.

\subsubsection{Statistical Analysis Methods and Reporting of the Results}

A variety of combined statistical methods for data analysis were adopted in the 29 selected studies for the current review. Parametric tests were typically used for between and within group statistical comparisons, including Analysis of Variance (ANOVA), Multivariate Analysis of Variance (MANOVA), Analysis of Covariance (ANCOVA), Independent-samples $T$ test, Paired-samples $T$ test, and One-sample $T$ test. To further probe the interaction effects, post hoc tests were conducted, including Tukey test and Student-Newman-Keuls test (SNK), and Bonferroni correction was generally adopted 
for multiple comparisons. Non-parametric tests were also administered for categorical variables in some studies, which included Chi-square test, Mann-Whitney U test (M-U) and Wilcoxon Rank test.

Approximately half of the studies (15 out of 29) calculated Pearson's and Spearman's rank-order correlation coefficients to examine the correlation between variables. Other studies also used more sophisticated approaches to address the relationships between multivariate measures, including multiple linear regression, multivariate linear regression and principal component analysis (PCA). In analyzing neurophysiological data [37,53,55], Statistical Parametric Mapping (SPM) appeared to be most frequently applied. Depending on the research hypotheses of the studies, laterality measures and voxel wise correlation approach were also used [32,37]. In general, $p<0.05$ was adopted for reporting statistical significance, and other significance levels with smaller alpha values such as 0.01 were also used.

\subsection{Performance of Emotional Prosody Processing}

All studies considered accuracy as the indicator of participants' behavioral performance of EPP. Five studies also recorded the response time for between group comparisons [35,37,38,41,55]. Among the studies with healthy participants as a control group, nearly all studies $(27 / 29)$ reached a consensus that schizophrenia patients (at least one of the schizophrenic patient groups) showed poorer performance than healthy controls except one study that tested audio-visual emotional integration [50] and another involving symptomatically remitted schizophrenic patients [11]. These two studies corresponded to the outliers in the right side of the funnel plot in Figure 2 with the mean value standing at 0 , indicating no significant difference between patients and healthy control in EPP performance. Analysis of schizophrenic patients' EPP impairment across the entire studies revealed a large overall effect size: $d=-0.92,95 \% \mathrm{CI}=-1.06<\delta<-0.78$. The effect size was larger for identification test paradigm: $d=-0.95,95 \% \mathrm{CI}=-1.11<\delta<-0.80$, but smaller for discrimination tasks $(d=-0.74$, $95 \% \mathrm{CI}=-1.03<\delta<-0.44)$. It is worth noticing that there was a study that only reported the results of comprehension tasks; thus, it was excluded from both identification and discrimination paradigm but included in the overall effect size calculation [28]. The overall meta-analysis results are presented in a funnel plot (Figure 2) and a forest plot (Figure 3).

There remained some inconsistent findings among studies dealing with different subtypes of schizophrenia (e.g., SAVH vs. SNAVH), the influence of which will be explained in the next section. Studies involving patients with other diseases suggested that patients with schizophrenia performed worse than those with depression [36], and their EPP were more statistically similar to RBD patients than LBD patients [28].

Less than half of the studies took recognition of single emotion into consideration, and even fewer studies analyzed the significant differences between groups from this perspective. The seven studies with between-group performance of single and overall emotion recognition are demonstrated in Tables 2 and 3. Although between-group recognition performance varied from one type of emotion to another, the most consistent result was the evidence of schizophrenic patients' more impairment in recognizing emotional stimuli than neutral ones [31,37,41,47].

Two studies further explored the misclassification of emotions during EPP tasks. Patients with auditory verbal hallucination were found to be more likely to misclassify happiness into fear and fear into sadness [31], while another study found that they were more likely to mislabel sadness into happiness [33]. However, Shea and his colleagues further pinpointed that patients without auditory verbal hallucination tended to misidentify sadness as neutrality, again indicating the differences associated with subtypes of schizophrenia. 
Table 2. EPP performance of participant groups (Identification task).

\begin{tabular}{|c|c|c|c|c|c|c|c|c|c|c|c|c|}
\hline \multirow{2}{*}{ Year } & \multirow{2}{*}{ First Author } & \multirow{2}{*}{$\begin{array}{l}\text { Effect Size of Performance in } \\
\text { Schizophrenic Patients as Compared } \\
\text { to Healthy Control (95\% CI) }\end{array}$} & \multirow[t]{2}{*}{ Task Paradigm and Material } & \multirow{2}{*}{$\begin{array}{l}\text { Type of Participants } \\
\text { (Number) }\end{array}$} & \multicolumn{6}{|c|}{ Single Emotion Recognition } & \multicolumn{2}{|c|}{$\begin{array}{c}\text { Overall Emotion } \\
\text { Recognition }\end{array}$} \\
\hline & & & & & Happy & Sad & Angry & Fearful & Surprised & Disgusted & Neutral & \\
\hline 2002 & Hooker & $d=-1.27(-1.91$ to -0.64$)$ & Identification (SSC) & SP (20)-HC (27) & & & & & & & & $\Delta$ \\
\hline 2005 & Leitman & $d=-1.65(-2.17$ to -1.13$)$ & Identification (SSC) & SP (43)-HC (34) & & & & & & & & $\Delta$ \\
\hline \multirow{2}{*}{2005} & \multirow{2}{*}{ Rossell } & \multirow{2}{*}{$d=-0.74(-1.25$ to -0.23$)$} & Identification 1 (SSC) & $\begin{array}{c}\text { SAVH (20)-HC (26) } \\
\text { SNAVH (20)-HC (26) } \\
\text { SAVH (20)-SNAVH (20) }\end{array}$ & $\bullet$ & $\vec{\Delta}$ & $\bullet$ & $\stackrel{\Delta}{\bullet}$ & & & & $\Delta$ \\
\hline & & & Identification 2 (SSC) & $\begin{array}{c}\text { SAVH (20)-HC (26) } \\
\text { SNAVH (20)-HC (26) } \\
\text { SAVH (20)-SNAVH (20) }\end{array}$ & $\bullet$ & $\Delta$ & $\Delta$ & & $\Delta$ & & $\because$ & 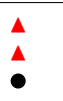 \\
\hline 2007 & Leitman & $d=-1.60(-2.32$ to -0.89$)$ & Identification (SSC) & SP (24)-HC (17) & & & & & & & & $\Delta$ \\
\hline 2007 & Shea & $d=-0.75(-1.18$ to -0.31$)$ & Identification (SSC) & $\begin{array}{l}\text { SAH (38)-HC (31) } \\
\text { SAH (38)-SNAH (29) } \\
\text { SNAH (29)-HC (31) }\end{array}$ & $\bullet$ & $\because$ & & & & & $\because$ & 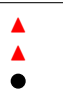 \\
\hline 2008 & Chan & $d=-1.40(-1.87$ to -0.93$)$ & Identification (SSC) & SP (43)-HC (43) & & & & & & & & $\Delta$ \\
\hline $2009 a$ & Bach & $d=-0.86(-1.44$ to -0.28$)$ & Identification (SPP) & $\begin{array}{c}\text { SP (25)-HC (25) } \\
\text { SP (25)-Depression (25) }\end{array}$ & $\bullet$ & $\bullet$ & $\bullet$ & $\bullet$ & & $\bullet$ & $\bullet$ & $\Delta$ \\
\hline $2009 b$ & Bach & $d=-0.94(-1.69$ to -0.18$)$ & Identification (SPP) & SP (15)-HC (15) & & & & & & & & $\Delta$ \\
\hline 2010 & Roux & $d=-0.66(-1.28$ to -0.04$)$ & Identification (SSC) & SP (21)-HC (21) & & & & & & & & $\Delta$ \\
\hline 2012 & Gold & $d=-0.79(-1.11$ to -0.47$)$ & $\begin{array}{l}\text { Identification (SPP-full } \\
\text { version) }\end{array}$ & SP (92)-HC (73) & $\bullet$ & $\Delta$ & $\bullet$ & $\Delta$ & & $\Delta$ & $\Delta$ & $\Delta$ \\
\hline 2013 & Jahshan & $d=-1.23(-1.90$ to -0.56$)$ & Identification (SSC) & SP (34)-HC (14) & $\bullet$ & $\bullet$ & $\bullet$ & $\bullet$ & & $\bullet$ & $\bullet$ & $\Delta$ \\
\hline 2013 & Kantrowitz & $d=-1.26(-1.73$ to -0.79$)$ & Identification (SSC and SPP) & SP (41)-HC (41) & & & & & & & & $\Delta$ \\
\hline 2013 & McLachlan & $d=-0.54(-1.13$ to 0.06$)$ & Identification (SSC) & SP (34)-HC (17) & & & & & & & & $\Delta$ \\
\hline 2013 & Pinheiro & $d=-0.70(-1.44$ to 0.04$)$ & $\begin{array}{l}\text { Identification (SSC) } \\
\text { Identification (SPP) }\end{array}$ & $\begin{array}{l}\text { SP (15)-HC (15) } \\
\text { SP (15)-HC (15) }\end{array}$ & $\bullet$ & & 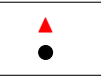 & & & & $\bullet$ & $\Delta$ \\
\hline 2013 & Tseng & $d=-1.07(-1.39$ to -0.75$)$ & Identification (SPP) & SP (111)-HC (70) & & & & & & & & $\Delta$ \\
\hline 2014 & Brazo & $d=-1.25(-2.01$ to -0.49$)$ & $\begin{array}{l}\text { Semantic identification } \\
\text { (EPP influences) }\end{array}$ & SP (16)-HC (16) & & & & & & & & $\Delta$ \\
\hline 2014 & Hoertnagl & $d=-0.04(-0.41$ to 0.33$)$ & $\begin{array}{l}\text { Identification (SSC) } \\
\text { Identification (SSC) }\end{array}$ & $\begin{array}{l}\text { SP (41)-BD (58) } \\
\text { SP (41)-HC (85) }\end{array}$ & $\bullet$ & $\bullet$ & $\bullet$ & $\bullet$ & & & & $\bullet$ \\
\hline 2014a & Kantrowitz & $d=-0.89(-1.23$ to -0.55$)$ & Identification (SSC) & SP (76)-HC (72) & & & & & & & & $\Delta$ \\
\hline $2014 \mathrm{~b}$ & Kantrowitz & $d=-1.22(-1.72$ to -0.72$)$ & $\begin{array}{l}\text { Identification (emotional } \\
\text { categories of SSC) }\end{array}$ & SP (31)-HC (44) & & & & & & & & $\Delta$ \\
\hline 2014 & Pinheiro & $d=-1.22(-1.98$ to -0.47$)$ & $\begin{array}{l}\text { Identification (SSC) } \\
\text { Identification (SPP) }\end{array}$ & $\begin{array}{l}\text { SP (16)-HC (16) } \\
\text { SP (16)-HC (16) }\end{array}$ & $\bullet$ & & 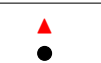 & & & & $\bullet$ & $\bullet$ \\
\hline
\end{tabular}


Table 2. Cont

\begin{tabular}{|c|c|c|c|c|c|c|c|c|c|c|c|c|c|}
\hline \multirow{2}{*}{ Year } & \multirow{2}{*}{ First Author } & \multirow{2}{*}{$\begin{array}{l}\text { Effect Size of Performance in } \\
\text { Schizophrenic Patients as Compared } \\
\text { to Healthy Control }(95 \% \mathrm{CI})\end{array}$} & \multirow{2}{*}{\multicolumn{2}{|c|}{ Task Paradigm and Material }} & \multirow{2}{*}{\multicolumn{2}{|c|}{$\begin{array}{l}\text { Type of Participants } \\
\text { (Number) }\end{array}$}} & \multicolumn{6}{|c|}{ Single Emotion Recognition } & \multirow{2}{*}{$\begin{array}{c}\begin{array}{c}\text { Overall Emotion } \\
\text { Recognition }\end{array} \\
\text { Neutral } \\
\end{array}$} \\
\hline & & & & & & & Happy & Sad & Angry & Fearful $\mathrm{S}$ & Surprised D & Disgusted & \\
\hline 2015 & Kantrowitz & $d=-0.91(-1.31$ to -0.51$)$ & \multicolumn{4}{|c|}{ Identification (SSC and SPP) } & & & & & & & $\Delta$ \\
\hline 2015 & Weisgerber & $d=-0.71(-1.23$ to -0.19$)$ & \multicolumn{2}{|c|}{ Identification (SPP) } & \multicolumn{2}{|c|}{ SP (30)-HC (30) } & $\bullet$ & $\bullet$ & $\bullet$ & $\bullet$ & $\bullet$ & $\bullet$ & $\bullet$ \\
\hline 2016 & Razafimandimby & $d=-0.51(-1.10$ to 0.08$)$ & \multicolumn{2}{|c|}{ Identification (SSC) } & \multicolumn{2}{|c|}{ SP (21)-HC (25) } & & & & & & & \\
\hline 2018 & Pawełczyk & $d=-1.16(-1.63$ to -0.68$)$ & Identification (SP & & SP (40)-HC & & & & & & & & $\Delta$ \\
\hline \multicolumn{14}{|c|}{$\begin{array}{l}\text { Note. (1) Negative effect size Cohen's d indicates worse performance in schizophrenic patients compared to healthy control; (2) Twenty-four out of } 29 \text { studies are reported in this table. } \\
\text { Other studies are not reported since they did not employ identification paradigm; (3) SSC = stimulus with semantic content; SPP = stimulus with pure prosody (without semantic } \\
\text { content); SP = schizophrenic patients; SAVH = schizophrenia with auditory verbal hallucination; } \mathrm{SAH}=\text { schizophrenia with auditory hallucination; SNAH = schizophrenia without } \\
\text { auditory hallucination; SNAVH = schizophrenia without auditory verbal hallucination; HC = healthy control; BD = patients with bipolar disorder; } \mathbf{\Lambda}=\text { Significant difference }(p<0.05) \text {; } \\
\bullet=\text { non-significant difference; blank = not evaluated; (4) The data were collected based on the accuracy rate reported in the included studies. }\end{array}$} \\
\hline \multirow{2}{*}{ Year } & \multirow{2}{*}{ First Author } & \multirow{2}{*}{$\begin{array}{c}\text { Effect Size of Performance in } \\
\text { Schizophrenic Patients as Compared } \\
\text { to Healthy Control }(95 \% \text { CI) }\end{array}$} & \multirow{2}{*}{$\begin{array}{l}\text { Task Paradigm and } \\
\text { Material }\end{array}$} & \multirow{2}{*}{\multicolumn{2}{|c|}{$\begin{array}{l}\text { Type of Participants } \\
\text { (Number) }\end{array}$}} & \multicolumn{7}{|c|}{ Single Emotion Recognition } & \multirow{2}{*}{$\begin{array}{c}\text { Overall Emotior } \\
\text { Recognition }\end{array}$} \\
\hline & & & & & & Happy & Sad & Angry & Fearful & Surprised & d Disgusted & d Neutral & \\
\hline 2005 & Leitman & $d=-1.64(-2.16$ to -1.12$)$ & $\begin{array}{l}\text { Discrimination } \\
\text { (SSC pair) }\end{array}$ & & 3)-HC (34) & & & & & & & & $\Delta$ \\
\hline 2005 & Rossell & $d=-0.72(-1.23$ to -0.21$)$ & $\begin{array}{l}\text { Discrimination } \\
\text { (dichotic listening } \\
\text { of SPP pair) }\end{array}$ & $\begin{array}{r}\text { SAV } \\
\text { SNA } \\
\text { SAVH }\end{array}$ & $\begin{array}{l}(20)-H C(26) \\
(20)-H C(26) \\
\text { )-SNAVH (20) }\end{array}$ & $\bullet$ & : & $\bullet$ & $\bullet$ & & & $\bullet$ & $\ddot{\Delta}$ \\
\hline 2007 & Leitman & $d=-1.34(-2.03$ to -0.65$)$ & $\begin{array}{l}\text { Discrimination } \\
\text { (SSC pair) }\end{array}$ & & 4)-HC (17) & & & & & & & & $\Delta$ \\
\hline 2008 & Chan & $d=-0.79(-1.23$ to -0.35$)$ & $\begin{array}{l}\text { Discrimination } \\
\text { (SSC pair) }\end{array}$ & & 3)-HC (43) & & & & & & & & $\Delta$ \\
\hline 2008 & Scholten & $d=-0.69(-1.11$ to -0.28$)$ & $\begin{array}{l}\text { Discrimination } \\
\text { (meaning-prosody } \\
\text { stroop test) }\end{array}$ & & 8)-HC (46) & & & & & & & & \\
\hline 2010 & Roux & $d=-0.45(-1.06$ to 0.16$)$ & $\begin{array}{l}\text { Discrimination } \\
\text { (meaning-prosody } \\
\text { stroop test) }\end{array}$ & & 1)-HC (21) & & & & & & & & $\Delta$ \\
\hline 2013 & Ito & $d=-0.44(-0.94$ to 0.05$)$ & $\begin{array}{l}\text { Discrimination } \\
\text { (meaning-prosody } \\
\text { stroop test) }\end{array}$ & & 8)-HC (37) & & & & & & & & $\Delta$ \\
\hline
\end{tabular}


Table 3. Cont

\begin{tabular}{|c|c|c|c|c|c|c|c|c|c|c|c|c|}
\hline \multirow{2}{*}{ Year } & \multirow{2}{*}{ First Author } & \multirow{2}{*}{$\begin{array}{c}\text { Effect Size of Performance in } \\
\text { Schizophrenic Patients as Compared } \\
\text { to Healthy Control }(95 \% \mathrm{CI})\end{array}$} & \multirow{2}{*}{$\begin{array}{c}\text { Task Paradigm and } \\
\text { Material }\end{array}$} & \multirow{2}{*}{$\begin{array}{l}\text { Type of Participants } \\
\text { (Number) }\end{array}$} & \multicolumn{7}{|c|}{ Single Emotion Recognition } & \multirow{2}{*}{$\begin{array}{l}\text { Overall Emotion } \\
\text { Recognition }\end{array}$} \\
\hline & & & & & Happy & Sad & Angry & Fearful & Surprised & Disgusted & Neutral & \\
\hline 2013 & Iwashiro & $d=-0.44(-1.02$ to 0.15$)$ & $\begin{array}{l}\text { Discrimination } \\
\text { (dichotic listening } \\
\text { of SSC pair) }\end{array}$ & SP (22)-HC (24) & & & & & & & & $\bullet$ \\
\hline 2014 & Müller & $d=-0.03(-0.75$ to 0.69$)$ & $\begin{array}{l}\text { Discrimination } \\
\text { (face-prosody } \\
\text { stroop test) }\end{array}$ & SP (15)-HC (15) & & & & & & & & $\bullet$ \\
\hline
\end{tabular}

Note. (1) Negative effect size Cohen's d indicates worse performance in schizophrenic patients compared to healthy control; (2) Nine out of 29 studies are reported in this table. Other studies

are not reported since they did not employ discrimination paradigm; (3) SSC = stimulus with semantic content; SPP = stimulus with pure prosody (without semantic content); SP = schizophrenic patients; $\mathrm{SAVH}=$ schizophrenia with auditory verbal hallucination; $\mathrm{SAH}=$ schizophrenia with auditory hallucination; $\mathrm{SNAH}=$ schizophrenia without auditory hallucination; $\mathrm{SNAVH}=$ schizophrenia without auditory verbal hallucination; $\mathrm{HC}=$ healthy control; $\boldsymbol{\Delta}=$ significant difference $(p<0.05)$; $\bullet=$ non-significant difference; blank = not evaluated; $(4)$ The data were collected based on the accuracy rate reported in the included studies. 


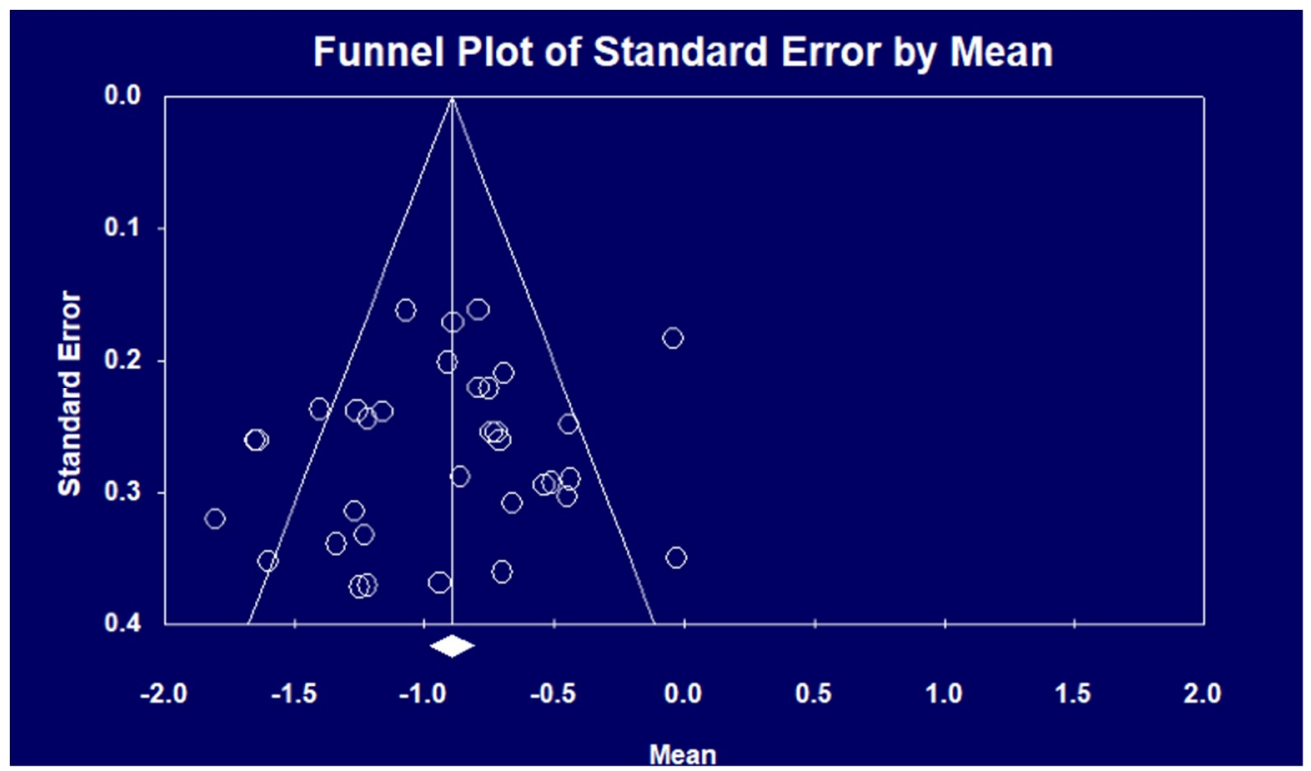

Figure 2. Funnel plot of the selected studies.
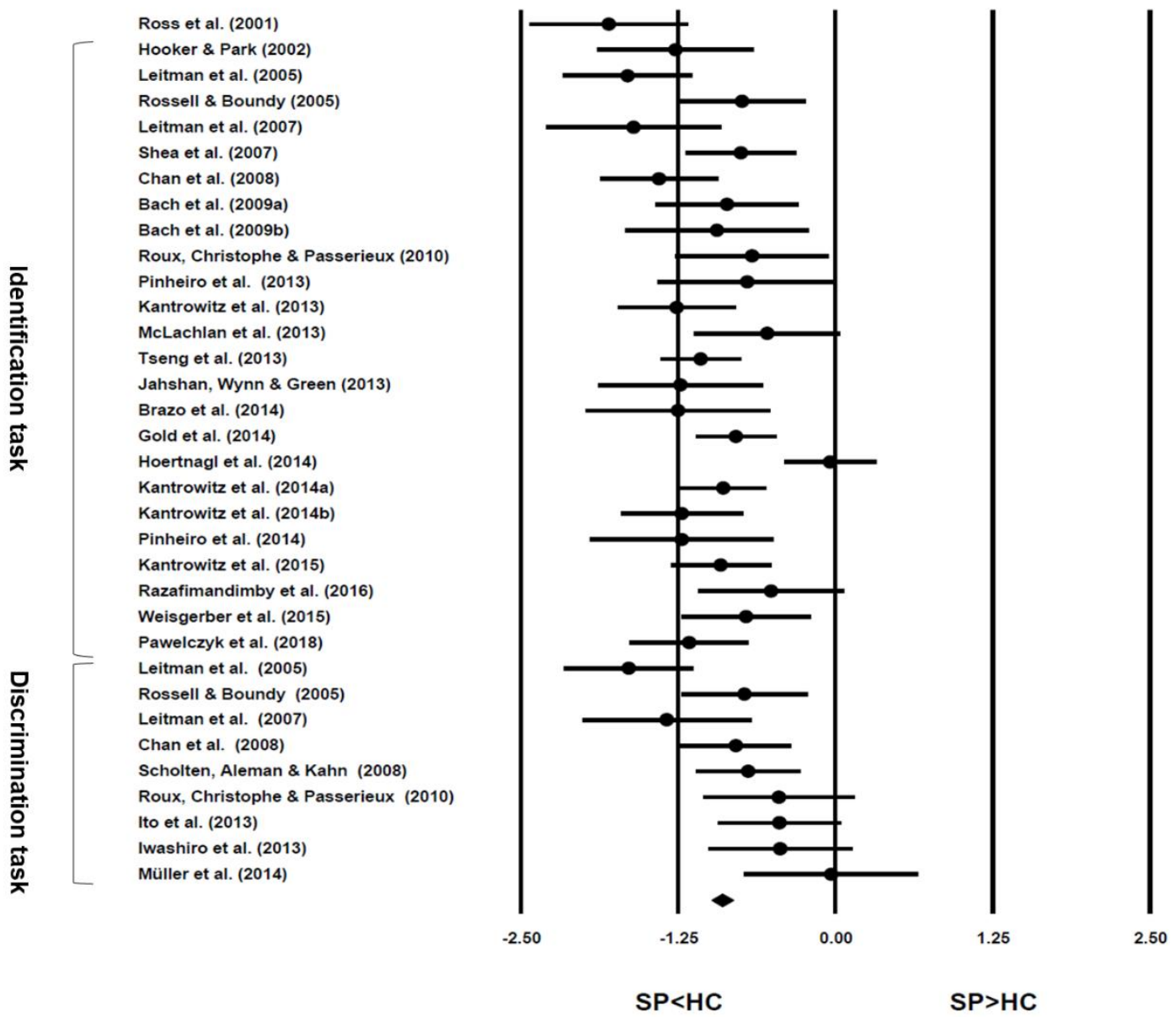

Figure 3. Forest plot with effect size $(d)$ and confidence intervals for the selected studies. Note: Ross et al.'s study (2001) only reported the results of comprehension tasks, thus excluded from the meta-analysis of both identification and discrimination paradigm but included in the overall effect size calculation. 


\subsection{Influential Factors of Emotional Prosody Processing}

The major influential factors that were reported by the selected studies can be categorized into two types: factors related to participants and factors related to experimental design.

\subsubsection{Factors Related to Participants}

Demographic Factors

Among the major demographic factors, selected studies found no differences that were attributable to IQ or education $[33,38]$. As for gender, a non-significant effect was identified in some studies $[30,36]$. However, female patients were found to preserve their advantages in EPP in another experiment [35], which explained the reason why social functioning in schizophrenic patients was less impaired in women than men.

\section{Cognitive Factors}

A series of studies delved into the cognitive factors influencing schizophrenic patients' EPP performance. Abnormal sensory processing, especially basic auditory processing of pitch and intensity, were significantly correlated with deficits in EPP $[30,42,45,53]$. Also, though it was claimed that no influence was exerted by attention in one study [33], more studies recognized both pre-attentive and attentive processing as important predictors of emotion recognition tasks $[34,41,42,53]$.

\section{Clinical Factors}

Using PANSS, several studies identified the negative correlation between the severity of schizophrenic illness and EPP performance $[38,46,50]$, but few studies discovered any difference made by the duration of diseases [33].

Positive symptom scores were found to contribute to a positive correlation with emotions of positive valence by more scholars $[40,41,46]$ with the exception of one study reporting no correlation [36]. Three of the selected studies also examined the effects of specific positive symptoms such as hallucination and delusion, which generally exacerbated schizophrenic patients' impairment in EPP $[33,41,45]$. However, this finding failed to be replicated when hallucinating patients were asked to identify the emotional prosody of semantically neutral sentences [44], indicating that EPP of schizophrenic patients was a rather complex process influenced by more than one factor.

Compared with positive symptoms, negative symptoms such as anhedonia were more consistently recognized to demonstrate a strong correlation with patient's deficits in EPP. Such a correlation was discovered with the aid of both behavioral and neurological research techniques $[30,35,36,38,43,46]$.

\subsubsection{Factors Related to Experiments}

\section{Task-Related Factors}

A small number of studies explored the effects of task types in schizophrenic patients' EPP [38]. The major finding was that compared with normal participants, schizophrenic patients had more difficulty in identification tasks than discrimination ones, and identifying the emotional prosody of the stimuli was even harder than emotional content [38].

Apart from emotional prosody tests, there were also studies (10 out of 29) including emotion recognition tasks in other modalities. This is not surprising as face-to-face interpersonal communication is inherently multimodal with both verbal and nonverbal signals, including tone of voice, facial expressions, hand gestures and body movements. However, the relationship among visual, vocal and musical modalities of test was still controversial. While facial expression identification was referred as a satisfactory predictor of emotional prosody in one study [36], more scholars discovered the disassociated processing of different modalities [29,30,34]. 


\section{Stimulus-Related Factors}

Among the stimulus-related factors, the emotional valence of stimuli had received most attention of the selected research. As suggested in the previous section, studies consistently found that patients with schizophrenia were more impaired in processing emotional stimuli than neutral ones $[31,37,40,41,50,51]$. It was further indicated that sadness was most difficult to detect for patients among all the emotions $[35,47]$.

The presence of semantic information, the congruency of emotional valence and the clarity of emotion were also proved to be influential factors of schizophrenic patient's EPP performance. Patients improved their performance when stimuli with semantic information were presented [51], and they showed an even better performance when semantic content and affective prosody of the stimuli shared a congruent emotional valence $[47,50]$. By contrast, patients were more likely to make an erroneous interpretation if they were asked to process low-clarity or potentially ambiguous emotions [29].

\subsection{Neural Mechanisms of Emotional Prosody Processing}

\subsubsection{Hemispheric Processing of Emotional Prosody}

Right hemisphere had been repeatedly reported dominant in EP processing for healthy controls $[63,64]$. The deficits in EPP suggested that the right hemispheric processing was disturbed for schizophrenic patients $[28,57]$. The right-lateralization decreased in patients with stronger negative symptoms and with hallucinating symptoms [37]. There was also a study suggesting left-lateralized abnormalities in schizophrenic patients, but it lacked neuroimaging data to support its conclusion [41].

\subsubsection{Spatial Localization of Brain Networks for Processing Emotional Prosody}

Three studies identified structural and functional disturbances of brain regions associated with the deficits in processing emotional prosody for schizophrenic patients by means of MRI tools. It was revealed that there might be dysfunctions in (a) primary auditory cortex [32] and (b) medial prefrontal cortex [55], both of which were important for social communication deficits. It was also identified reduced auditory-insula connectivity as a determinant of social cognitive dysfunction in schizophrenic patients [53].

\subsubsection{Temporal Processing of Emotional Prosody}

The neural correlates for examining the time course of EPP in the selected ERP studies included mismatched negativity (MMN), P50, P1, N100, P200 and P300. Significant reduction in MMN indicated an impaired pre-attentive processing in schizophrenia [42,53]. A reduced P300 and a significant correlation between MMN and P300 within the patient group were found, which demonstrated that pre-attentive (MMN) and later attention-dependent processes (P300) contributed generally equally to EP change detection in patients with schizophrenia [42].

Other neural correlates investigated in the selected studies were mainly associated with sensory processing of the emotional valence of the stimuli. For example, there were also reduced P1, P50 and N100 when patients were processing emotionally incongruent stimuli, happy stimuli and neutral stimuli respectively $[45,50,51]$. However, increased P200 was observed when happy stimuli were presented, which was correlated with delusions [45,51].

\section{Discussion}

Overall, despite differences across studies in the current selective review, there is a general pattern of significantly worse performance of EPP in schizophrenic patients compared with healthy controls with large effect sizes as shown in our meta-analysis, which is consistent with the previous review [24]. Our analysis further revealed a more severe impairment in perceiving EP in identification 
tasks. Moreover, the studies consistently found a significant association between EPP and participants' cognitive and clinical factors such as auditory processing deficits, the severity and negative symptoms of the disease. The cognitive brain research studies further demonstrated that schizophrenic patients had EPP dysfunctions in both pre-attentive and attentive cognitive processes, which can be attributed to disorders in right hemisphere and brain regions such as medial prefrontal cortex, primary auditory cortex and auditory-insula connectivity. However, some of the findings involving stimuli of different modalities, participants of different genders, language backgrounds and subtypes as well as positive symptoms of schizophrenia, remained controversial. The convergent and divergent findings have important implications for clinical practice and research.

\subsection{Implications of the Selected Studies}

\subsubsection{Implications for Practice}

One of the most salient findings from this review, is that schizophrenic patients generally had poorer performance in processing emotional prosody. To date, emotional prosody has been recognized as an important window to detect schizophrenic deficits, and it has also played a role in improving people's social communication $[47,50]$. The feasibility and efficacy of EPP intervention have been demonstrated in several studies. For example, a recent systematic review and meta-analysis concluded that future clinical trials should consider applying a cognition remediation program that combines social cognition training elements and psychiatric rehabilitation [14]. An empirical study also demonstrated that schizophrenic patients were able to improve their affective prosody perception while being involved in community-based psychosocial rehabilitation programs [13]. Cognitive ability such as melodic discrimination and the factors influencing cognition such as acoustic features of the stimuli were also valued as crucial targets of cognitive remediation in schizophrenia $[39,49]$. However, to our knowledge, few programs have incorporated it into the early diagnosis and remediation of schizophrenic disorders [38]. Current clinical classifications including both the revised version of the WHO International Classification of Disorders (ICD-11) and the Diagnostic and Statistical Manual of Mental Disorders (DSM-5) by the American Psychiatric Association also did not specify EPP as a target area for assessment or treatment $[65,66]$, although the DSM-5 classification system does include functional impairment and the ICD-11 encourages the use of functional impairment as diagnostic criteria. The strong effect sizes across the reviewed studies provide the most telling evidence that emotional prosody, either in verbal or non-verbal forms (e.g., natural sounds, music), should be implemented as an important supplemental clinical indicator in the early detection of schizophrenia. For example, it is possible to better distinguish patients with schizophrenia from healthy controls by observing their EP performance in perceptual tasks with emotional stimuli of different modalities and in identification paradigms. Furthermore, a milder approach could be combined with clinical medication in the rehabilitation of schizophrenia. For example, a line of studies pinpointed the role of bottom-up cognitive remediation paradigms [42,43,53]. Apart from sensory-cognitive methods, it is advisable to consider employment of other behavioral therapeutic approaches of psychology and speech pathology, in which EP can be an important component.

\subsubsection{Implications for Research}

There are some apparent limitations of the selected studies. First, none of the included research works were longitudinal studies, which failed to provide data tracing patients' performance over time. Schizophrenia is known to be a psychiatric disorder that varies in severity with a time course of single or multiple psychotic episodes potentially leading to life-long disability. The short-term and long-term characteristics in symptomology may show different trajectories of relapses and remissions for positive and negative symptoms along with the treatment time course and medication history. Similarly, cognitive functioning such as executive functions, attentional control, and working memory is known to show age-related differences and changes. However, none of the studies covered in the 
current review delved into symptomology changes and changes in overall cognition in relation to the patients' EPP ability. Second, only two of the 29 studies addressed the production of schizophrenic patients' emotional prosody so that production and perception data could not be directly compared across the studies. As difficulties in distinguishing self and other is at the core of schizophrenia, it remains an important question for researchers to explore the exact forms of relationship between EP comprehension and production in these patients. Third, the number of studies conducted in different languages was imbalanced, with non-tonal languages outnumbering tonal ones and stressed-timed languages outnumbering syllable-timed ones.

More cross-sectional and longitudinal studies are needed to investigate the behavioral and neuro-cognitive mechanisms of schizophrenic patients' perception and production of emotional prosody. There are at least three new directions for future studies in this regard.

First, more studies conducted in tonal languages (e.g., Mandarin Chinese) and syllable-timed languages (e.g., Mandarin Chinese and French) are called for in this field [34]. More importantly, there needs to be more cross-linguistic investigation of the relationship between emotional prosody and linguistic units extending from phonetics and semantics to morphology, grammar, syntax and pragmatics. The process of verbal communication may vary from not only speaker to speaker, but also from language to language. The selected studies did not employ the same tasks to compare patients with different ethnic, language and cultural backgrounds, and to associate EP perception with their EP production, thus rendering the cross-language generalization difficult. Moreover, as an important component of extralinguistic domains, prosody is closely connected with other linguistic domains in understanding speaker's meaning. Thus, new directions for research will surely benefit from new perspectives such as schizophrenic patients' cross-linguistic and cross-cultural communication differences in EP perception and production.

Secondly, given the existing controversial views about emotional recognition of different modalities, the influences of different sensory domains are worthy of further exploration. Currently, a line of studies has discovered schizophrenic patients' deficits in processing emotional stimuli not only in vocal modalities but also in visual forms [67]. The disassociation among different modalities in understanding emotions was also found [29,30,34]. However, the reasons for the disassociated results may lie in that these studies tended to separate cross-modal processing of emotion in different tasks rather than in the same one, in which visual and vocal stimuli were presented simultaneously [50]. By contrast, studies of face-voice emotion integration in normal subjects have shown strong facilitation effects in terms of accuracy, sensitivity, and reaction time [68]. De Silva et al. demonstrated that in normal subjects, some emotions such as sadness and fear in videos are better identified in the auditory modality whereas other emotions such as anger and happiness are better recognized in the visual modality [69]. A recent EEG study on normal subjects further revealed visual-auditory priming effects in distinct neural oscillatory activities for emotional prosody processing as against phonetic processing [70]. It remains to be tested how patients with schizophrenia differ from normal individuals in such multimodal experimental paradigms. Only by involving different forms of stimuli (such as visual, auditory, and tactile information) in the same task can we truly test the interactions in multisensory integration, which is required of daily functions in face-to-face social interactions.

Finally, cognition also serves as a promising testbed for researching schizophrenic patients' comprehension of emotional prosody. It has been discovered that both pre-attentive and attentive processing play significant roles in emotion recognition tasks [34,41,42,53]. Future studies concerning emotional prosody understanding can be extended to other neuro-cognitive domains such as memory, monitory, thoughts and reasoning, literacy, language production and problem-solving abilities. It remains to be explored how EPP deficits may be linked with genetics, brain chemistry and specific brain circuits (or information processing pathways), neuroanatomical abnormality, and environmental factors. Furthermore, as EPP deficits were identified in other psychotic disorders such as borderline personality disorder [16] and autism spectrum disorder [17], similarities and differences in behaviors, 
neurocognitive structures and functions, and genetic representations among different types of patients deserve in-depth investigation, which may lay a more solid foundation for effective intervention of schizophrenia and for the exploration of brain plasticity in future research [71]. Despite the similarities on behavioral and psychological measures of social cognition deficits that may be shared across different types of clinical patients including schizophrenia, it remains to be investigated whether their behavioral symptomology may reveal subtle distinctions and how those can be traced down and linked with distinct underlying neural mechanisms and genetic epidemiology.

\subsection{Limitations of This Selective Review}

Our own analysis in this review bears several limitations. First, our review was limited to peer-reviewed publications which were hypothesis-driven research studies, and because of the large quantity of studies during the searching process, there might be a few missing reports even though we strived to be careful when including the studies. Second, as the sample size and comprehensiveness of neuropsychological profiles of the patients varied widely in the studies, caution is necessary to interpret the results from different subtypes of schizophrenic patients, different task paradigms and response patterns, and stimuli of different modalities, emotional valence and presentation forms. The breadth of types of studies may also pose obstacles to generalizing some of the results. Due to space consideration, our classification of identification vs. discrimination tasks and characterization of the materials and details of the experimental protocols are likely oversimplified in the summary description. Finally, the selection criteria could serve as a potential source of bias in estimating the overall significance of EPP deficits in schizophrenic patients. In our search and selection criteria, we excluded patients with co-morbidity and prodromal schizophrenia. We originally intended to narrow down the scope of participants and provide a more accurate description of patients who only suffered from schizophrenia at the time of the experiments, but this may, to some extent, render our selective review disconnected from the real-world diagnosis, prevention, and remediation of schizophrenia. In this regard, some recent studies have touched upon schizophrenic patients at various stages of the disease such as prodromal schizophrenia [52], first-episode schizophrenia [56], and chronic schizophrenia [48] as well as with different co-morbid disorders such as depression $[18,56]$ and schizoaffective disorders [19], which may be enlightening for future empirical research.

\section{Conclusions}

The present study provides a selective review of the literature on emotional prosody processing in schizophrenic patients. A total of 29 behavioral and neural studies with great variability in research topics, experimental design and study participants were selected and summarized. Compared with healthy controls, schizophrenic patients showed worse performance of EPP, especially in identification tasks involving emotional and neutral stimuli than in discrimination tasks. Apart from experiment-related factors, the EPP deficits were generally found to be associated with cognitive and clinical factors such as auditory processing deficits, the severity and negative symptoms of the disease, while demographic factors such as IQ and education proved to make little difference. Neural evidence indicated impairments in patients' right hemisphere and dysfunctions in primary auditory cortex, medial prefrontal cortex, and auditory-insula connectivity, which provided a biological explanation for their social cognitive dysfunction. Moreover, the patients showed impaired pre-attentive and attentive processes, both of which played important roles in their EPP. The current review supports the use of EP assessment in early diagnosis and rehabilitation of schizophrenic disorders in clinical practice. Future cross-sectional and longitudinal study topics are further suggested to gain insights about schizophrenic patient's perception and production of EP in different languages and cultures, modality forms and neuro-cognitive domains.

Funding: This research was funded by the interdisciplinary program of Shanghai Jiao Tong University grant number (14JCZ03). The APC was funded by the interdisciplinary program of Shanghai Jiao Tong University grant number (14JCZ03). 
Acknowledgments: Zhang was supported by a summer visiting professorship from Shanghai Jiao Tong University to work on the project. We also thank the anonymous reviewers and the editors for providing us with insightful suggestions to revise our review.

Conflicts of Interest: The authors declare no conflict of interest. The funders had no role in the design of the study; in the collection, analyses, or interpretation of data; in the writing of the manuscript, and in the decision to publish the results.

\section{References}

1. World Health Organization. Schizophrenia Fact Sheet. 2018. Available online: www.who.int/news-room/ fact-sheets / detail/schizophrenia (accessed on 15 October 2018).

2. Shenton, M.E.; Dickey, C.C.; Frumin, M.; McCarley, R.W. A review of MRI findings in schizophrenia. Schizophr. Res. 2001, 49, 1-52. [CrossRef]

3. Wible, C.G.; Preus, A.P.; Hashimoto, R. A cognitive neuroscience view of schizophrenic symptoms: Abnormal activation of a system for social perception and communication. Brain Imaging Behav. 2009, 3, 85-110. [CrossRef] [PubMed]

4. Edwards, J.; Jackson, H.J.; Pattison, P.E. Emotion recognition via facial expression and affective prosody in schizophrenia: A methodological review. Clin. Psychol. Rev. 2002, 22, 789-832. [CrossRef]

5. Mueser, K.T.; Doonan, R.; Penn, D.L.; Blanchard, J.J.; Bellack, A.S.; Nishith, P.; DeLeon, J. Emotion recognition and social competence in chronic schizophrenia. J. Abnorm. Psychol. 1996, 105, 271-275. [CrossRef] [PubMed]

6. Penn, D.L.; Sanna, L.J.; Roberts, D.L. Social cognition in schizophrenia: An overview. Schizophr. Bull. 2008, 34, 408-411. [CrossRef] [PubMed]

7. Pinkham, A.E.; Penn, D.L.; Perkins, D.O.; Lieberman, J. Implications for the neural basis of social cognition for the study of schizophrenia. Am. J. Psychiatry 2003, 160, 815-824. [CrossRef] [PubMed]

8. Fett, A.K.J.; Viechtbauer, W.; Penn, D.L.; van Os, J.; Krabbendam, L. The relationship between neurocognition and social cognition with functional outcomes in schizophrenia: A meta-analysis. Neurosci. Biobehav. Rev. 2011, 35, 573-588. [CrossRef] [PubMed]

9. Sterea, R. The relationship between social cognition and functional outcomes in schizophrenia. Procedia Soc. Behav. Sci. 2015, 187, 256-260. [CrossRef]

10. Penn, D.L.; Spaulding, W.; Reed, D.; Sullivan, M. The relationship of social cognition to ward behavior in chronic schizophrenia. Schizophr. Res. 1996, 20, 327-335. [CrossRef]

11. Hoertnagl, C.M.; Yalcin-Siedentopf, N.; Baumgartner, S.; Biedermann, F.; Deisenhammer, E.A.; Hausmann, A.; Kaufmann, A.; Kemmler, G.; Mühlbacher, M.; Rauch, A.; et al. Affective prosody perception in symptomatically remitted patients with schizophrenia and bipolar disorder. Schizophr. Res. 2014, 158, 100-104. [CrossRef] [PubMed]

12. Varga, E.; Herold, R.; Tényi, T.; Bugya, T. Social Cognition Analyzer Application (SCAN)—A new approach to analyse social cognition in schizophrenia. Eur. Neuropsychopharm. 2018, 28, S55-S56. [CrossRef]

13. Varga, E.; Endre, S.; Bugya, T.; Tényi, T.; Herold, R. Community-based psychosocial treatment has an impact on social processing and functional outcome in schizophrenia. Front. Psychiatry 2018, 9, 247. [CrossRef] [PubMed]

14. Revell, E.R.; Neill, J.C.; Harte, M.; Khan, Z.; Drake, R.J. A systematic review and meta-analysis of cognitive remediation in early schizophrenia. Schizophr. Res. 2015, 168, 213-222. [CrossRef] [PubMed]

15. Alba-Ferrara, L.; De Erausquin, G.A.; Hirnstein, M.; Weis, S.; Hausmann, M. Emotional prosody modulates attention in schizophrenia patients with hallucinations. Front. Hum. Neurosci. 2013, 7, 59. [CrossRef] [PubMed]

16. Minzenberg, M.J.; Poole, J.H.; Vinogradov, S. Social-emotion recognition in borderline personality disorder. Compr. Psychiatry 2006, 47, 468-474. [CrossRef] [PubMed]

17. Rosenblau, G.; Kliemann, D.; Dziobek, I.; Heekeren, H.R. Emotional prosody processing in autism spectrum disorder. Soc. Cogn. Affect. Neurosci. 2017, 12, 224-239. [CrossRef] [PubMed]

18. Regenbogen, C.; Kellermann, T.; Seubert, J.; Schneider, D.A.; Gur, R.E.; Derntl, B.; Schneider, F.; Habel, U. Neural responses to dynamic multimodal stimuli and pathology-specific impairments of social cognition in schizophrenia and depression. Br. J. Psychiatry 2015, 206, 198-205. [CrossRef] [PubMed] 
19. Kantrowitz, J.T.; Hoptman, M.J.; Leitman, D.I.; Silipo, G.; Javitt, D.C. The 5\% difference: Early sensory processing predicts sarcasm perception in schizophrenia and schizoaffective disorder. Psychol. Med. 2014, 44, 25-36. [CrossRef] [PubMed]

20. Haskins, B.; Shutty, M.S.; Kellogg, E. Affect processing in chronically psychotic patients: Development of a reliable assessment tool. Schizophr. Res. 1995, 15, 291-297. [CrossRef]

21. Edwards, J.; Pattison, P.E.; Jackson, H.J.; Wales, R.J. Facial affect and affective prosody recognition in first-episode schizophrenia. Schizophr. Res. 2001, 48, 235-253. [CrossRef]

22. Kucharska-Pietura, K.; David, A.S.; Masiak, M.; Phillips, M.L. Perception of facial and vocal affect by people with schizophrenia in early and late stages of illness. Br. J. Psychiatry 2005, 187, 523-528. [CrossRef] [PubMed]

23. Pinkham, A.E.; Penn, D.L. Neurocognitive and social cognitive predictors of interpersonal skill in schizophrenia. Psychiatry Res. 2006, 143, 167-178. [CrossRef] [PubMed]

24. Hoekert, M.; Kahn, R.S.; Pijnenborg, M.; Aleman, A. Impaired recognition and expression of emotional prosody in schizophrenia: Review and meta-analysis. Schizophr. Res. 2007, 96, 135-145. [CrossRef] [PubMed]

25. Ekman, P. An Argument for Basic Emotions. Cogn. Emot. 1992, 6, 169-200. [CrossRef]

26. World Health Organization. The ICD-10 Classification of Mental and Behavioural Disorders: Clinical Description and Diagnostic Guidelines; World Health Organization: Geneva, Switzerland, 1992; pp. 84-97.

27. Kay, S.R.; Fiszbein, A.; Opler, L.A. The positive and negative syndrome scale (PANSS) for schizophrenia. Schizophr. Bull. 1987, 13, 261-276. [CrossRef] [PubMed]

28. Ross, E.D.; Orbelo, D.M.; Cartwright, J.; Hansel, S.; Burgard, M.; Testa, J.A.; Buck, R. Affective-prosodic deficits in schizophrenia: Profiles of patients with brain damage and comparison with relation to schizophrenic symptoms. J. Neurol. Neurosurg. Psychiatry 2001, 70, 597-604. [CrossRef] [PubMed]

29. Hooker, C.; Park, S. Emotion processing and its relationship to social functioning in schizophrenia patients. Psychiatry Res. 2002, 112, 41-50. [CrossRef]

30. Leitman, D.I.; Foxe, J.J.; Butler, P.D.; Saperstein, A.; Revheim, N.; Javitt, D.C. Sensory contributions to impaired prosodic processing in schizophrenia. Biol. Psychiatry 2005, 58, 56-61. [CrossRef] [PubMed]

31. Rossell, S.L.; Boundy, C.L. Are auditory-verbal hallucinations associated with auditory affective processing deficits? Schizophr. Res. 2005, 78, 95-106. [CrossRef] [PubMed]

32. Leitman, D.I.; Hoptman, M.J.; Foxe, J.J.; Saccente, E.; Wylie, G.R.; Nierenberg, J.; Jalbrzikowski, M.; Lim, K.O.; Javitt, D.C. The neural substrates of impaired prosodic detection in schizophrenia and its sensorial antecedents. Am. J. Psychiatry 2007, 164, 474-482. [CrossRef] [PubMed]

33. Shea, T.L.; Sergejew, A.A.; Burnham, D.; Jones, C.; Rossell, S.L.; Copolov, D.L.; Egan, G.F. Emotional prosodic processing in auditory hallucinations. Schizophr. Res. 2007, 90, 214-220. [CrossRef] [PubMed]

34. Chan, C.; Wong, R.; Wang, K.; Lee, T. Emotion recognition in Chinese people with schizophrenia. Psychiatry Res. 2008, 157, 67-76. [CrossRef] [PubMed]

35. Scholten, M.R.; Aleman, A.; Kahn, R.S. The processing of emotional prosody and semantics in schizophrenia: Relationship to gender and IQ. Psychol. Med. 2008, 38, 887-898. [CrossRef] [PubMed]

36. Bach, D.R.; Buxtorf, K.; Grandjean, D.; Strik, W.K. The influence of emotion clarity on emotional prosody identification in paranoid schizophrenia. Psychol. Med. 2009, 39, 927-938. [CrossRef] [PubMed]

37. Bach, D.R.; Herdener, M.; Grandjean, D.; Sander, D.; Seifritz, E.; Strik, W.K. Altered lateralisation of emotional prosody processing in schizophrenia. Schizophr. Res. 2009, 110, 180-187. [CrossRef] [PubMed]

38. Roux, P.; Christophe, A.; Passerieux, C. The emotional paradox: Dissociation between explicit and implicit processing of emotional prosody in schizophrenia. Neuropsychologia 2010, 48, 3642-3649. [CrossRef] [PubMed]

39. Gold, R.; Butler, P.; Revheim, N.; Leitman, D.I.; Hansen, J.A.; Gur, R.C.; Kantrowitz, J.T.; Laukka, P.; Juslin, P.N.; Silipo, G.S.; et al. Auditory emotion recognition impairments in schizophrenia: Relationship to acoustic features and cognition. Am. J. Psychiatry 2012, 169, 424-432. [CrossRef] [PubMed]

40. Ito, F.; Matsumoto, K.; Miyakoshi, T.; Ohmuro, N.; Uchida, T.; Matsuoka, H. Emotional processing during speech communication and positive symptoms in schizophrenia. Psychiatry Clin. Neurosci. 2013, 67, 526-531. [CrossRef] [PubMed]

41. Iwashiro, N.; Yahata, N.; Kawamuro, Y.; Kasai, K.; Yamasue, H. Aberrant interference of auditory negative words on attention in patients with schizophrenia. PLoS ONE 2013, 8, e83201. [CrossRef] [PubMed]

42. Jahshan, C.; Wynn, J.K.; Green, M.F. Relationship between auditory processing and affective prosody in schizophrenia. Schizophr. Res. 2013, 143, 348-353. [CrossRef] [PubMed] 
43. Kantrowitz, J.T.; Leitman, D.I.; Lehrfeld, J.M.; Laukka, P.; Juslin, P.N.; Butler, P.D.; Silipo, G.; Javitt, D.C. Reduction in tonal discriminations predicts receptive emotion processing deficits in schizophrenia and schizoaffective disorder. Schizophr. Bull. 2013, 39, 86-93. [CrossRef] [PubMed]

44. McLachlan, N.M.; Phillips, D.S.; Rossell, S.L.; Wilson, S.J. Auditory processing and hallucinations in schizophrenia. Schizophr. Res. 2013, 150, 380-385. [CrossRef] [PubMed]

45. Pinheiro, A.P.; Del, R.E.; Mezin, J.; Nestor, P.G.; Rauber, A.; Mccarley, R.W.; Gonçalves, O.F.; Niznikiewicz, M.A. Sensory-based and higher-order operations contribute to abnormal emotional prosody processing in schizophrenia: An electrophysiological investigation. Psychol. Med. 2013, 43, 603-618. [CrossRef] [PubMed]

46. Tseng, H.H.; Chen, S.H.; Liu, C.M.; Howes, O.; Huang, Y.L.; Hsieh, M.H.; Liu, C.C.; Shan, J.C.; Lin, Y.T.; $\mathrm{Hwu}, \mathrm{H}$.G. Facial and prosodic emotion recognition deficits associate with specific clusters of psychotic symptoms in schizophrenia. PLoS ONE 2013, 8, e66571. [CrossRef] [PubMed]

47. Brazo, P.; Beaucousin, V.; Lecardeur, L.; Razafimandimby, A.; Dollfus, S. Social cognition in schizophrenic patients: The effect of semantic content and emotional prosody in the comprehension of emotional discourse. Front. Psychiatry 2014, 5, 120. [CrossRef] [PubMed]

48. Dondaine, T.; Robert, G.; Péron, J.; Grandjean, D.; Vérin, M.; Drapier, D.; Millet, B. Biases in facial and vocal emotion recognition in chronic schizophrenia. Front. Psychol. 2014, 5, 900. [CrossRef] [PubMed]

49. Kantrowitz, J.T.; Scaramello, N.; Jakubovitz, A.; Lehrfeld, J.M.; Laukka, P.; Elfenbein, H.A.; Silipo, G.; Javitt, D.C. Amusia and protolanguage impairments in schizophrenia. Psychol. Med. 2014, 44, 2739-2748. [CrossRef] [PubMed]

50. Müller, V.I.; Kellermann, T.S.; Seligman, S.C.; Turetsky, B.I.; Eickhoff, S.B. Modulation of affective face processing deficits in schizophrenia by congruent emotional sounds. Soc. Cogn. Affect. Neurosci. 2014, 9, 436-444. [CrossRef] [PubMed]

51. Pinheiro, A.P.; Rezaii, N.; Rauber, A.; Liu, T.; Nestor, P.G.; Mccarley, R.W.; Gonçalves, O.F.; Niznikiewicz, M.A. Abnormalities in the processing of emotional prosody from single words in schizophrenia. Schizophr. Res. 2014, 152, 235-241. [CrossRef] [PubMed]

52. Corcoran, C.M.; Keilp, J.G.; Kayser, J.; Klim, C.; Butler, P.D.; Bruder, G.E.; Gur, R.C.; Javitt, D.C. Emotion recognition deficits as predictors of transition in individuals at clinical high risk for schizophrenia: A neurodevelopmental perspective. Psychol. Med. 2015, 45, 2959-2973. [CrossRef] [PubMed]

53. Kantrowitz, J.T.; Hoptman, M.J.; Leitman, D.I.; Morenoortega, M.; Lehrfeld, J.M.; Dias, E.; Sehatpour, P.; Laukka, P.; Silipo, G.; Javitt, D.C. Neural substrates of auditory emotion recognition deficits in schizophrenia. J. Neurosci. 2015, 35, 14909-14921. [CrossRef] [PubMed]

54. Weisgerber, A.; Vermeulen, N.; Peretz, I.; Samson, S.; Philippot, P.; Maurage, P.; De Graeuwe D'Aoust, C.; De Jaegere, A.; Delatte, B.; Gillain, B.; et al. Facial, vocal and musical emotion recognition is altered in paranoid schizophrenic patients. Psychiatry Res. 2015, 229, 188-193. [CrossRef] [PubMed]

55. Razafimandimby, A.; Hervé, P.Y.; Marzloff, V.; Brazo, P.; Tzourio-Mazoyer, N.; Dollfus, S. Functional deficit of the medial prefrontal cortex during emotional sentence attribution in schizophrenia. Schizophr. Res. 2016, 178, 86-93. [CrossRef] [PubMed]

56. Herniman, S.E.; Allott, K.A.; Killackey, E.; Hester, R.; Cotton, S.M. The effect of comorbid depression on facial and prosody emotion recognition in first-episode schizophrenia spectrum. J. Affect. Disord. 2017, 208, 223-229. [CrossRef] [PubMed]

57. Pawełczyk, A.; Kotlickaantczak, M.; Łojek, E.; Ruszpel, A.; Pawełczyk, T. Schizophrenia patients have higher-order language and extralinguistic impairments. Schizophr. Res. 2018, 192, 274-280. [CrossRef] [PubMed]

58. Wilson, D. B. Practical meta-analysis effect size calculator. Available online: http://www. campbellcollaboration.org/escalc/html/EffectSizeCalculator-SMD-main.php (accessed on 15 October 2018).

59. Borenstein, M.; Hedges, L.V.; Higgins, J.P.T.; Rothstein, H.R. Comprehensive Meta-Analysis 2.0; Biostat: Englewood, NJ, USA, 2005.

60. Borenstein, M.; Hedges, L.V.; Higgins, J.P.T.; Rothstein, H.R. A basic introduction to fixed-effect and random-effects models for meta-analysis. Res. Synth. Methods 2010, 1, 97-111. [CrossRef] [PubMed]

61. Cohen, J. Statistical Power Analysis for the Behavioral Sciences, 2nd ed.; Lawrence Earlbaum Associates: Hillsdale, NJ, USA, 1988; pp. 40-41. ISBN 0-8058-0283-5. 
62. Juslin, P.N.; Laukka, P. Impact of intended emotion intensity on cue utilization and decoding accuracy in vocal expression of emotion. Emotion 2001, 1, 381-412. [CrossRef] [PubMed]

63. Ross, E.D.; Thompson, R.D.; Yenkosky, J. Lateralization of affective prosody in brain and the callosal integration of hemispheric language functions. Brain Lang. 1997, 56, 27-54. [CrossRef] [PubMed]

64. Wildgruber, D.; Riecker, A.; Hertrich, I.; Erb, M.; Grodd, W.; Ethofer, T.; Ackermann, H. Identification of emotional intonation evaluated by fMRI. Neuroimage 2005, 24, 1233-1241. [CrossRef] [PubMed]

65. World Health Organization. ICD-11 for Mortality and Morbidity Statistics. Available online: https://icd. who.int/browse11/1-m/en (accessed on 15 October 2018).

66. American Psychiatric Association. Diagnostic and Statistical Manual of Mental Disorders, 5th ed.; American Psychiatric Publishing: Arlington, WA, USA, 2013; pp. 1-51.

67. Kohler, C.G.; Walker, J.B.; Martin, E.A.; Healey, K.M.; Moberg, P.J. Facial emotion perception in schizophrenia: A meta-analytic review. Schizophr. Bull. 2009, 36, 1009-1019. [CrossRef] [PubMed]

68. Massaro, D.W.; Egan, P.B. Perceiving affect from the voice and the face. Psychon. Bull. Rev. 1996, 3, $215-221$. [CrossRef] [PubMed]

69. De Silva, L.C.; Miyasato, T.; Nakatsu, R. Facial Emotion Recognition Using Multimodal Information. In Proceedings of the IEEE International Conference on Information, Communications and Signal Processing (ICICS'97), Singapore, 9-12 September 1997; pp. 397-401.

70. Diamond, E.; Zhang, Y. Cortical processing of phonetic and emotional information in speech: A cross-modal priming study. Neuropsychologia 2016, 82, 110-122. [CrossRef] [PubMed]

71. Kingdon, D.G.; Ashcroft, K.; Bhandari, B.; Gleeson, S.; Warikoo, N.; Symons, M.; Taylor, L.; Lucas, E.; Mahendra, R.; Ghosh, S.; et al. Schizophrenia and borderline personality disorder: Similarities and differences in the experience of auditory hallucinations, paranoia, and childhood trauma. J. Nerv. Ment. Dis. 2010, 198, 399-403. [CrossRef] [PubMed]

(C) 2018 by the authors. Licensee MDPI, Basel, Switzerland. This article is an open access article distributed under the terms and conditions of the Creative Commons Attribution (CC BY) license (http:/ / creativecommons.org/licenses/by/4.0/). 